\title{
S-Almost Automorphic Solutions for Impulsive Evolution Equations on Time Scales in Shift Operators
}

\author{
Chao Wang ${ }^{1}{ }^{\circledR}$, Rathinasamy Sakthivel ${ }^{2, *}$ and Gaston M. N'Guérékata ${ }^{3}$ \\ 1 Department of Mathematics, Yunnan University, Kunming 650091, Yunnan, China; chaowang@ynu.edu.cn \\ 2 Department of Applied Mathematics, Bharathiar University, Coimbatore, Tamil Nadu 641046, India \\ 3 Department of Mathematics, Morgan State University, 1700 E. Cold Spring Lane, Baltimore, MD 21251, USA; \\ Gaston.NGuerekata@morgan.edu \\ * Correspondence: krsakthivel@yahoo.com
}

Received: 1 April 2020; Accepted: 18 June 2020; Published: 23 June 2020

check for updates

\begin{abstract}
In this paper, based on the concept of complete-closed time scales attached with shift direction under non-translational shifts (or S-CCTS for short), as a first attempt, we develop the concepts of $S$-equipotentially almost automorphic sequences, discontinuous S-almost automorphic functions and weighted piecewise pseudo $S$-almost automorphic functions. More precisely, some novel results about their basic properties and some related theorems are obtained. Then, we apply the introduced new concepts to investigate the existence of weighted piecewise pseudo $S$-almost automorphic mild solutions for the impulsive evolution equations on irregular hybrid domains. The obtained results are valid for $q$-difference partial dynamic equations and can also be extended to other dynamic equations on more general time scales. Finally, some heat dynamic equations on various hybrid domains are provided as applications to illustrate the obtained theory.
\end{abstract}

Keywords: S-almost automorphic functions; impulsive evolution equations; mild solutions; time scales

MSC: 43A60; 34N05; 34A36; 26E70

\section{Introduction}

Almost automorphic functions, which are more general than the almost periodic functions, were introduced by Bochner (see [1-3]) in relation to some aspects of differential geometry. Almost automorphic solutions in the context of differential equations have been studied by several researchers. For instance, pseudo and weighted pseudo almost automorphic mild solutions to (fractional) evolution equations were investigated by Chang et al. [4-6], Ding et al. [7,8], Diagana [9,10]. Subsequently some interesting properties of the space of weighted pseudo almost automorphic functions like the completeness and the composition theorem were reported in [11,12] by N'Guérékata which have many applications in the context of differential equations. For more details about this topic we refer to the recent books (see $[10,11]$ ), where the authors gave important overviews about the theory of almost automorphic functions and their applications to differential equations.

Since time-scale calculus was proposed by Hilger [13], Bohner and Guseinov have extensively developed this theory on the aspect of integral and dynamic equations (see $[14,15])$. To study the approximation properties of time scales, some new concepts such as almost periodic time scales and changing-periodic time scales were proposed and studied by Agarwal et al. (see [16,17]). In addition to these fundamental results, there have been many works on different types of dynamic equations on time scales. For example, the concept of variable time scales was introduced and a novel idea of the mutual transformation between impulsive dynamic equations and dynamic equations on variable 
time scales was initiated by Akhmet et al. [18-20]. In the literature [21], Bohner et al. established an SIR model on the general time scales and derived its exact solution. In [22], the existing ideas of the univariate case of the time-scale calculus was generalized to the bivariate case and applied to partial dynamic equations. In the stability analysis, Martynyuk and Stamova investigated the sets of dynamic equations and hybrid dynamic systems on time scales (see $[23,24])$. In $[25,26]$, two types of new high order derivations were introduced and the existence of solutions for the type high order fractional integro-differential equations was studied by Aydogan and Baleanu et al., and these types of fractional corresponding derivatives were generalized to time scales by Mozyrska, Ortigueira and Torres et al. (see $[27,28]$ ). In the field of studying functions and applications, it is a hot topic to study the almost automorphic and almost periodic functions and applications to dynamic equations based on time scales. For example, Hong investigated the almost periodic set-valued functions and almost periodic set dynamic equations on time scales (see [29]). On almost automorphic functions and its related problems, Kéré, Mophou, N'Guérékata et al. investigated (n-order) almost automorphic and asymptotically almost automorphic functions of $n$-order, some basic results were obtained and applied to abstract dynamic equations (see [30-32]). In 2020, based on the concepts the authors introduced on translation time scales, Wang et al. established a theory of closedness of translation time scales and their applications to evolution equations and dynamical models (see the monograph [33]). In addition, a new concept of periodic time scales and the notion of shift operators of time scales were proposed and studied under the background of studying periodic functions (see Adivar et al. [34,35]). It is easy to observe that periodic time scales under translations have a nice closedness and their graininess function $\mu$ is bounded.

However, some classical and important time scales are irregular and they have the unbounded graininess function $\mu$. For example, $\mathbb{T}=\overline{q^{\mathbb{N}}}:=\left\{q^{t}: t \in \mathbb{N}_{0}\right.$ for $\left.q>1\right\} \cup\{0\}$, where $\mathbb{N}_{0}$ is the

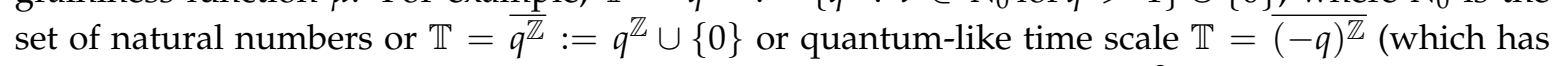
applications in quantum theory) and other types of time scales like $\mathbb{T}=\mathbb{N}^{2}$ and $\mathbb{T}=\mathbb{T}_{n}$ the space of the harmonic numbers (it is of interest to study almost automorphic dynamic behavior of solutions for q-difference-like dynamic equations among others, see Wang et al. [36-38]). It is impossible to introduce almost automorphic functions on such a type of time scale since the translation approximation of functions will never be reached for the reason that the graininess function $\mu$ is a strictly increasing function for time scales. In addition, many natural phenomena must be modeled as a process in which continuous evolution is usually interrupted by an event (impulses, catastrophe, etc., see Stamova [39,40] and Wang et al. [41-43]), which motivates us to investigate general evolution equations with impulses on irregular hybrid domains.

In the present paper, for the first time, we study the existence of weighted piecewise pseudo $S$-almost automorphic mild solutions for the impulsive evolution dynamic equations

$$
\left\{\begin{array}{l}
x^{\Delta}(t)=A(t) x^{\sigma}+f(t, x(t)), t \in \mathbb{T}, t \neq t_{k}, k \in \mathbb{Z}, \\
\widetilde{\Delta} x\left(t_{k}\right)=x\left(t_{k}^{+}\right)-x\left(t_{k}^{-}\right)=I_{k}\left(x\left(t_{k}\right)\right), t=t_{k}
\end{array}\right.
$$

where $A \in P C_{r d}(\mathbb{T}, B(\mathbb{X}))$ is a bounded linear operator in the Banach space $\mathbb{X}$ and $f \in P C_{r d}(\mathbb{T} \times$ $\mathbb{X}, \mathbb{X}), x^{\sigma}=x(\sigma(t)) . f, I_{k}, t_{k}$ satisfy suitable conditions that will be established later and $\mathbb{T}$ is a complete-closed time scale attached with shift direction under non-translational shifts (S-CCTS). In addition, the notations $x\left(t_{k}^{+}\right)$and $x\left(t_{k}^{-}\right)$represent the right-hand and the left-hand side limits of $x(\cdot)$ at $t_{k}$, respectively. In addition, some Lemmas are obtained and the exponential stability of weighted piecewise pseudo $S$-almost automorphic mild solutions is also studied. Finally, we apply these obtained results to study a class of $\Delta$-partial differential equations on $S$-CCTS. The obtained results in this paper are feasible and effective on $q$-difference partial dynamic equations and more.

For instance, in (1), by using the shift operators $\delta_{ \pm}$in Section 2, 
(i) if we let $\mathbb{T}=\mathbb{N}^{\frac{1}{2}}=\{\sqrt{n}: n \in \mathbb{N}\}$ and $t_{k}=\sqrt{k^{2}+1}, k \in \mathbb{Z}$, then (1) turns into

$$
\left\{\begin{array}{l}
\frac{x\left(\sqrt{t^{2}+1}\right)-x(t)}{\sqrt{t^{2}+1}-t}=A(t) x^{\sigma}+f(t, x(t)), t \in \mathbb{T}, t \neq t_{k}, k \in \mathbb{Z} \\
\widetilde{\Delta}\left(t_{k}\right)=x\left(t_{k}^{+}\right)-x\left(t_{k}^{-}\right)=I_{k}\left(x\left(t_{k}\right)\right), t=t_{k}
\end{array}\right.
$$

(ii) if we let $\mathbb{T}=\overline{\left\{q^{n}: q>1, n \in \mathbb{Z}\right\}}=\overline{q^{\mathbb{Z}}}$ and $t_{k}=q^{k^{3}}, k \in \mathbb{Z}$, then (1) turns into

$$
\left\{\begin{array}{l}
\frac{x(q t)-x(t)}{(q-1) t}=A(t) x^{\sigma}+f(t, x(t)), t \in \mathbb{T}, t \neq t_{k}, k \in \mathbb{Z}, \\
\widetilde{\Delta} x\left(t_{k}\right)=x\left(t_{k}^{+}\right)-x\left(t_{k}^{-}\right)=I_{k}\left(x\left(t_{k}\right)\right), t=t_{k}
\end{array}\right.
$$

(iii) if we let $\mathbb{T}=h \mathbb{Z}, h>0$ and $t_{k}=h k^{3}, k \in \mathbb{Z}$, then (1) turns into

$$
\left\{\begin{array}{l}
\frac{x(t+h)-x(t)}{h}=A(t) x^{\sigma}+f(t, x(t)), t \in \mathbb{T}, t \neq t_{k}, k \in \mathbb{Z}, \\
\widetilde{\Delta} x\left(t_{k}\right)=x\left(t_{k}^{+}\right)-x\left(t_{k}^{-}\right)=I_{k}\left(x\left(t_{k}\right)\right), t=t_{k}
\end{array}\right.
$$

(iv) if we let $\mathbb{T}=\left\{(-q)^{n}: q>1, n \in \mathbb{Z}\right\} \cup\{0,1\}$ and $t_{k}=(-q)^{3 k}, k \in \mathbb{Z}$, then (1) turns into

$$
\left\{\begin{array}{l}
\frac{x\left(q_{t} t\right)-x(t)}{\left(q_{t}-1\right) t}=A(t) x^{\sigma}+f(t, x(t)), t \in \mathbb{T}, t \neq t_{k}, k \in \mathbb{Z}, \\
\widetilde{\Delta} x\left(t_{k}\right)=x\left(t_{k}^{+}\right)-x\left(t_{k}^{-}\right)=I_{k}\left(x\left(t_{k}\right)\right), t=t_{k},
\end{array}\right.
$$

where $q_{t}=q^{2}$ if $t>0$ and $q_{t}=1 / q^{2}$ if $t<0$, it is a classical $q$-dynamic system on quantum-like hybrid domains.

We provide four types of impulsive evolution dynamic equations in the above, in fact, (1) will turn into other different types of dynamic equations on different types of complete-closed time scales attached with shift direction under translational or non-translational shifts.

The highlights of the paper can be summarized as follows

- We introduce the concept of $S$-equipotentially almost automorphic sequences under $S$-CCTS.

- We establish a theory of discontinuous $S$-almost automorphic functions and weighted piecewise pseudo $S$-almost automorphic functions. Some new results about their basic properties and some related theorems are obtained.

- The existence of weighted piecewise pseudo $S$-almost automorphic mild solutions for the impulsive evolution equations on irregular hybrid domains is studied.

- The obtained results in this paper are effective for $q$-difference heat equations and other dynamic equations on more general hybrid domains.

\section{S-Equipotentially Almost Automorphic Sequence Under S-CCTS}

In this section, we will introduce some knowledge of complete-closed time scales under non-translational shifts (or S-CCTS for short) and then define S-equipotentially almost automorphic sequence and study its properties. For more details about time-scale calculus and S-CCTS, one may refer to the book $[14,17]$.

For convenience, we introduce the notations. Let $\mathbb{T}^{*}$ be the largest open subset of $\mathbb{T}$, i.e., $\overline{\mathbb{T}^{*}}=\mathbb{T}$.

$$
\mathcal{D}_{ \pm}=\left\{(s, t) \in \mathbb{T}^{*} \times \mathbb{T}^{*}: \delta_{ \pm}(s, t) \in \mathbb{T}^{*}\right\} .
$$

For any $s \in \mathbb{T}^{*}$, denote

$$
\mathbb{T}_{*^{-}}^{\delta_{s^{-}}}:=\delta_{-}\left(s, \mathbb{T}^{*}\right):=\left\{\delta_{-}(s, t):(s, t) \in \mathcal{D}_{-}, \forall t \in \mathbb{T}^{*}\right\},
$$




$$
\mathbb{T}_{*}^{\delta_{\delta^{+}}}:=\delta_{+}\left(s, \mathbb{T}^{*}\right):=\left\{\delta_{+}(s, t):(s, t) \in \mathcal{D}_{+}, \forall t \in \mathbb{T}^{*}\right\} .
$$

Definition 1 ([36]). Let $\mathbb{T}$ be a time scale with the shift operators $\delta_{ \pm}$associated with the initial point $t_{0} \in \mathbb{T}^{*}$. The time scale $\mathbb{T}$ is said to be bi-direction S-CCTS in shifts $\delta_{ \pm}$if

$$
\Pi^{ \pm}:=\left\{p \in \mathbb{T}^{*}:(p, t) \in \mathcal{D}_{ \pm} \text {for all } t \in \mathbb{T}^{*}\right\} \notin\left\{\left\{t_{0}\right\}, \varnothing\right\} .
$$

Remark 1. Note that from (2) and (3), we obtain that (4) can be written into the equivalent form $\Pi^{ \pm}=\{p \in$ $\left.\mathbb{T}^{*}: \mathbb{T}_{*}^{\delta^{ \pm}} \subseteq \mathbb{T}^{*}\right\} \notin\left\{\left\{t_{0}\right\}, \varnothing\right\}$.

Example 1. According to Definition 1, we provide the following examples of S-CCTS. Let $\mathbb{T}=\overline{(-q)^{\mathbb{Z}}}=$ $\left\{(-q)^{n}: q>1, n \in \mathbb{Z}\right\} \cup\{0\}$. We can obtain that $\Pi^{ \pm}=\left\{(-q)^{2 n}: q>1, n \in \mathbb{Z}\right\}$. For such a time scale, for any $t \in \mathbb{T}^{*}$, take $t_{0}=1$, we attach the shift operators

$$
\delta_{+}(s, t)=\left\{\begin{array}{l}
s t, t>0, \\
\frac{t}{s}, t<0,
\end{array} \quad \delta_{-}(s, t)=\left\{\begin{array}{l}
\frac{t}{s}, t>0 \\
s t, t<0 .
\end{array}\right.\right.
$$

Hence, there exists $q^{2} \in \Pi^{ \pm}$such that $\delta_{ \pm}\left(q^{2}, t\right) \in \mathbb{T}^{*}$ for all $t \in \mathbb{T}^{*}$, i.e., $\Pi^{ \pm} \notin\{\{1\}, \varnothing\}$. From Definition 1 , $\mathbb{T}$ is a S-CCTS with bi-direction.

Remark 2. Note that if $\mathbb{T}$ is a periodic time scales under translations and $\Pi^{ \pm} \subseteq \mathbb{T}^{*}$, then the shift operators will fulfill $\delta_{ \pm}(\tau, t)=t \pm \tau \in \mathbb{T}$ with the initial point $t_{0}=0$. Hence, if $\Pi^{ \pm} \subseteq \mathbb{T}^{*}$, then $T$-CCTS is included in S-CCTS.

If $\mathbb{T}$ is a bi-direction S-CCTS and $t_{0}$ is the initial point, then for any $s \in \Pi^{ \pm}$, we define a function $A: \Pi^{ \pm} \rightarrow \Pi^{ \pm}$,

$$
A(s)= \begin{cases}\delta_{+}\left(s, t_{0}\right), & s>t_{0}, \\ \delta_{-}\left(s, t_{0}\right), & s<t_{0},\end{cases}
$$

which will be used later. Note that $A(s)>t_{0}$ and $A(s) \geq s$.

Remark 3. If $\Pi^{ \pm} \subset \mathbb{T}^{*}$ and $\delta_{ \pm}\left(s, t_{0}\right)=s \pm t_{0}$, let $t_{0}=0$, then one can easily obtain $A(s)=|s|$.

In what follows, we will demonstrate some examples to show the almost automorphic phenomena for functions on S-CCTS, which are completely different from the cases on periodic time scales under translations.

Example 2. Let $\mathbb{T}=\mathbb{R}$ and $\Pi=[0,+\infty)$, and we define the following operators:

$$
\delta_{+}(\tau, t)=\left\{\begin{array}{ll}
\tau t, & \text { if } t \geq 0, \\
t / \tau, & \text { if } t<0,
\end{array} \quad \text { for } \tau \in[1,+\infty) \cap \Pi,\right.
$$

and

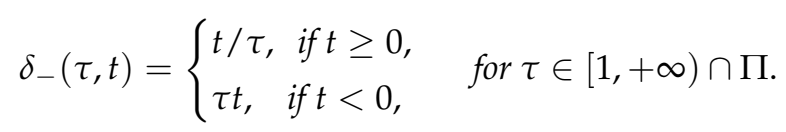

Step 1. Periodic function construction. We know that the set of reals $\mathbb{R}$ is periodic under shifts $\delta_{ \pm}$. The function

$$
f_{\tau}(t)=\cos \left(\frac{\ln |t|}{\ln (1 / \sqrt{\tau})} \pi\right), \tau>1 \text { and } t \in \mathbb{T}^{*}=\mathbb{R} \backslash\{0\}
$$


is periodic under shifts $\delta_{ \pm}$with the period $\tau=P^{2}, P>1$ since

$$
\begin{aligned}
& f_{\tau}\left(\delta_{\tau^{ \pm 1}}(t)\right)=\left\{\begin{array}{ll}
f_{\tau}\left(t P^{ \pm 2}\right), & \text { if } t \geq 0, \\
f_{\tau}\left(t / P^{ \pm 2}\right), & \text { if } t<0,
\end{array} \quad=\cos \left(\frac{\ln |t| \pm 2 \ln (1 / P)}{\ln (1 / P)} \pi\right)\right. \\
& =\cos \left(\frac{\ln |t|}{\ln (1 / P)} \pi \pm 2 \pi\right)=\cos \left(\frac{\ln |t|}{\ln (1 / P)} \pi\right)=f_{\tau}(t) .
\end{aligned}
$$

Step 2. Almost periodic function construction. Based on Step 1, consider the function

$$
\tilde{F}(t)=\cos \left(\frac{\ln |\sqrt{2} t|}{\ln \left(1 / P_{1}\right)} \pi\right)+\cos \left(\frac{\ln |\sqrt{3} t|}{\ln \left(1 / P_{2}\right)} \pi\right)
$$

where $P_{1} \neq P_{2}, P_{1}, P_{2}>1$ and $t \in \mathbb{T}^{*}=\mathbb{R} \backslash\{0\}$. One can observe that $\tilde{F}(t)$ is almost periodic under shifts $\delta_{ \pm}$. From Step 1, let

$$
f_{P_{1}^{2}}(\sqrt{2} t)=\cos \left(\frac{\ln |\sqrt{2} t|}{\ln \left(1 / P_{1}\right)} \pi\right), f_{P_{2}^{2}}(\sqrt{3} t)=\cos \left(\frac{\ln |\sqrt{3} t|}{\ln \left(1 / P_{2}\right)} \pi\right),
$$

we obtain that $\tilde{F}(t)=f_{P_{1}^{2}}(\sqrt{2} t)+f_{P_{2}^{2}}(\sqrt{3} t)$, and note that $f_{P_{1}^{2}}$ and $f_{P_{2}^{2}}$ are periodic with different periods $P_{1}^{2}, P_{2}^{2}$, respectively.

Step 3. S-almost automorphic function construction. According to the above, consider the function

$$
\hat{F}(t)=\cos \left(\frac{1}{2+\left[\cos \left(\frac{\ln |\sqrt{2} t|}{\ln \left(1 / P_{1}\right)} \pi\right)+\cos \left(\frac{\ln |\sqrt{3} t|}{\ln \left(1 / P_{2}\right)} \pi\right)\right]}\right),
$$

where $P_{1} \neq P_{2}, P_{1}, P_{2}>1$ and $t \in \mathbb{T}^{*}=\mathbb{R} \backslash\{0\}$. One can observe that $\hat{F}(t)$ is almost automorphic under shifts $\delta_{ \pm}$. From Step 2, we can obtain that $\hat{F}(t)=\cos \left(\frac{1}{2+f_{P_{1}^{2}}(\sqrt{2} t)+f_{P_{2}^{2}}(\sqrt{3} t)}\right)$.

Example 3. The time scale $\overline{q^{\mathbb{Z}}}=\left\{q^{n}: n \in \mathbb{Z}\right.$ and $\left.q=\sqrt[3]{3}\right\} \cup\{0\}$ is periodic with period $\tau$ under the shift operator $\delta_{ \pm}$.

Step 1. Periodic function construction. The piecewise periodic function defined by

$$
f_{1}(t)=[\theta(t)]^{\frac{\ln t}{\ln q}}, f_{2}(t)=[\theta(t)]^{\frac{\ln q t}{\ln q}}, \theta(t)= \begin{cases}1, & t>1, \\ -1, & 0<t<1 .\end{cases}
$$

Let $\tau=q^{4}$ and $\delta_{ \pm}(\tau, t)=q^{ \pm 4} t \in \overline{q^{\mathbb{Z}}} \backslash\{0\}=q^{\mathbb{Z}}$, we have $f_{1}\left(\delta_{ \pm}(\tau, t)\right)=[\theta(t)]^{\frac{\ln t}{\ln q} \pm 4}=[\theta(t)]^{\frac{\ln t}{\ln q}}=f_{1}(t)$ for all $t \in q^{\mathbb{Z}}$. Hence, $f_{1}(t)$ is a periodic function with period $q^{4}$. Similarly, let $\tau=q^{5}$, we can obtain $f_{2}\left(\delta_{ \pm}(\tau, t)\right)=f_{2}(t)$.

Step 2. Almost periodic function construction.

Through Step 1, we can obtain an almost periodic piecewise function

$$
F(t)=f_{1}(t)+f_{2}(t)=[\theta(t)]^{\frac{\ln t}{\ln q}}+[\theta(t)]^{\frac{\ln q t}{\ln q}}
$$

on $q^{\mathbb{Z}}$, where $f_{1}, f_{2}$ are periodic piecewise functions on $q^{\mathbb{Z}}$, respectively. Note that the periods of $f_{1}$ and $f_{2}$ are completely different.

Step 3. Almost automorphic function construction.

According to the above, let

$$
\tilde{F}(t)=\frac{1}{3+F(t)}=\frac{1}{3+[\theta(t)]^{\frac{\ln t}{\ln q}}+[\theta(t)]^{\frac{\ln q t}{\ln q}}}
$$


which is an almost automorphic function on $\overline{q^{\mathbb{Z}}}$.

Let

$$
\mathbb{B}=\left\{\left\{t_{k}\right\}: t_{k} \in \mathbb{T}: t_{k+1}>t_{k}, k \in \mathbb{Z}, \lim _{k \rightarrow \pm \infty} t_{k}= \pm \infty\right\}
$$

with $\theta=\inf _{k \in \mathbb{Z}} \delta_{-}\left(t_{k}, t_{k+1}\right)>0$.

Next, based on Definition 1, we will introduce the concept of S-equipotentially almost automorphic sequence and study its properties.

Definition 2. Assume $\mathbb{T}$ is S-CCTS with shifts $\delta_{ \pm}$. Let $\left\{t_{k}\right\} \in \mathbb{B}, k \in \mathbb{Z}$. We say $\left\{t_{k}^{j}\right\}$ is a S-derivative sequence of $\left\{t_{k}\right\}$ and

$$
t_{k}^{j}=\delta_{-}\left(t_{k}, t_{k+j}\right), k, j \in \mathbb{Z}
$$

Remark 4. If $\mathbb{T}$ is a periodic time scale under translations, then one can obtain the classical derivative sequence of $\left\{t_{k}\right\}$ satisfying $t_{k}^{j}=t_{k+j}-t_{k}$ by letting $\delta_{ \pm}(s, t)=t \pm s$. Particularly, if $\mathbb{T}=\mathbb{R}$, one can obtain the derivative sequence of $\left\{t_{k}\right\}$ from [40] (pp. 191-194) immediately.

Lemma 1 ([37]). If $\mathbb{T}^{*}$ be the largest subset of $\mathbb{T}$ and including a fixed number $t_{0} \in \mathbb{T}^{*}$ such that there exist operators $\delta_{ \pm}: \mathbb{T}^{*} \rightarrow \mathbb{T}^{*}$, then

(1) $\delta_{-}\left(s, \delta_{+}(u, t)\right)=\delta_{+}\left(\delta_{-}(s, u), t\right), \quad \delta_{+}\left(s, \delta_{-}(u, t)\right)=\delta_{-}\left(\delta_{-}(s, u), t\right)$.

(2) $\delta_{-}\left(s, \delta_{-}(u, t)\right)=\delta_{-}\left(\delta_{+}(s, u), t\right), \quad \delta_{+}\left(s, \delta_{+}(u, t)\right)=\delta_{+}\left(\delta_{+}(s, u), t\right)$.

(3) $\delta_{+}\left(\delta_{-}(u, s), \delta_{-}(s, t)\right)=\delta_{-}\left(\delta_{+}(u, s), \delta_{+}(s, t)\right)=\delta_{-}(u, t)$.

Lemma 2 ([37]). If $t_{k}^{j}=\delta_{-}\left(t_{k}, t_{k+j}\right)$ and $k, j \in \mathbb{Z}$, then

$$
\delta_{-}\left(t_{k}^{j}, t_{k+k_{1}}^{j}\right)=\delta_{-}\left(t_{k}^{k_{1}}, t_{k+j}^{k_{1}}\right), \delta_{-}\left(t_{k}^{k_{1}}, t_{k}^{j}\right)=t_{k+k_{1}}^{j-k_{1}} .
$$

Based on the S-derivative sequence and its properties, we will propose the following definition.

Definition 3. Let $\mathbb{T}$ be a S-CCTS under shifts $\delta_{ \pm}$and $t_{k}^{j}=\delta_{-}\left(t_{k}, t_{k+j}\right), k, j \in \mathbb{Z}$. The sequence $\left\{t_{k}^{j}\right\}, k, j \in \mathbb{Z}$ is said to be S-equipotentially almost automorphic if for any sequence $\left\{s_{n}\right\} \subset \mathbb{Z}$, there exists a subsequence $\left\{s_{n}^{\prime}\right\}$ such that

$$
\lim _{n \rightarrow \infty} t_{k}^{s_{n}^{\prime}}=\gamma_{k}
$$

is well defined for each $k \in \mathbb{Z}$ and

$$
\lim _{n \rightarrow \infty} \gamma_{k}^{-s_{n}^{\prime}}=t_{k}
$$

for each $k \in \mathbb{Z}$.

Remark 5. In Definition 3, note that $\mathbb{T}$ is a closed subset of $\mathbb{R}$, thus, for each $k \in \mathbb{Z}$, one has $\gamma_{k} \in \mathbb{T}$.

\section{S-Almost Automorphic Functions and Weighted Pseudo S-Almost Automorphic Functions}

Let $\mathbb{X}$ be a Banach space endowed with the norm $\|\cdot\| . B(\mathbb{X}, \mathbb{Y})$ denotes the Banach space of all bounded linear operators from $\mathbb{X}$ to $\mathbb{Y}$. This is simply denoted as $B(\mathbb{X})$ when $\mathbb{X}=\mathbb{Y}$. $B C(\mathbb{T}, \mathbb{X})$ is the space of bounded continuous function from $\mathbb{T}$ to $\mathbb{X}$ equipped with the supremum norm defined by $\|u\|_{\infty}=\sup _{t \in \mathbb{T}}\|u(t)\|$.

In the following, we will give the definition of $r d$-piecewise continuous functions on time scales.

Definition 4. We say $\varphi: \mathbb{T} \rightarrow \mathbb{X}$ is $r d$-piecewise continuous with respect to a sequence $\left\{t_{k}\right\} \subset \mathbb{T}$ which satisfy $t_{k}<t_{k+1}, k \in \mathbb{Z}$, if $\varphi(t)$ is continuous on $\left[t_{k}, t_{k+1}\right)_{\mathbb{T}}$ and $r d$-continuous on $\mathbb{T} \backslash\left\{t_{k}\right\}$. Furthermore, $\left[t_{k}, t_{k+1}\right)_{\mathbb{T}}$ are called intervals of continuity of the function $\varphi(t)$. 
For convenience, $P C_{r d}(\mathbb{T}, \mathbb{X})$ denotes the set of all $r d$-piecewise continuous functions with respect to a sequence $\left\{t_{k}\right\}, k \in \mathbb{Z}$. For $\left\{t_{k}\right\}_{k \in \mathbb{Z}} \in \mathbb{B}$, let

$$
\begin{aligned}
& B P C_{r d}(\mathbb{T}, \mathbb{X})=:\left\{\phi \in P C_{r d}(\mathbb{T}, \mathbb{X}):\right. \\
& \left.\|\phi\| \leq M, \text { where } M \text { is a positive constant and } \phi\left(t_{k}\right)=\phi\left(t_{k}^{+}\right), k \in \mathbb{Z}\right\}
\end{aligned}
$$

let $\Omega$ be a subset of $\mathbb{X}$ and

$$
\begin{aligned}
& B P C_{r d}(\mathbb{T} \times \Omega, \mathbb{X})=:\left\{\phi \in P C_{r d}(\mathbb{T} \times \Omega, \mathbb{X}):\|\phi\| \leq M, \text { where } M\right. \text { is a positive constant } \\
& \text { and } \left.\phi\left(t_{k}, x\right)=\phi\left(t_{k}^{+}, x\right), k \in \mathbb{Z}, \phi(t, \cdot) \text { is continuous at } x \in \Omega \text { for any } t \in \mathbb{T}\right\} \\
& \qquad P C(\mathbb{T}, \mathbb{X})=:\left\{\varphi \in P C_{r d}(\mathbb{T}, \mathbb{X}):\right. \\
& \left.\varphi \text { is uniformly rd-continuous on the interval }\left[t_{k}, t_{k+1}\right)_{\mathbb{T}} \text { for } k \in \mathbb{Z}\right\} .
\end{aligned}
$$

Remark 6. $\varphi \in C_{r d}(\mathbb{T}, \mathbb{X})$ is uniformly $r d$-continuous on the interval $[a, b]_{\mathbb{T}}$ if and only if for any $\varepsilon>0$, there exists $\delta(\varepsilon)>0$ such that for all right dense points $t_{1}, t_{2} \in \mathbb{T}$ and $\left|t_{1}-t_{2}\right|<\delta$ implies $\left\|\varphi\left(t_{1}\right)-\varphi\left(t_{2}\right)\right\|<\varepsilon$ (see Definition 2.1 of [29]).

Now, let $T, P \in \mathbb{B}$ and let $s(T \cup P): \mathbb{B} \rightarrow \mathbb{B}$ be a map such that the set $s(T \cup P)$ forms a strictly increasing sequence. For $D \subset \mathbb{T}$ and $v>0$, we introduce the notations $\theta_{v}(D)=\left\{\delta_{-}(v, t), t \in D\right\}$, $F_{v}(D)=D \cap\left\{\theta_{v}(D)\right\}$. Denote by $\tilde{\phi}=(\varphi(t), T)$ the element from the space $P C_{r d}(\mathbb{T}, \mathbb{X}) \times \mathbb{B}$. For every sequence of real numbers $\left\{s_{n}\right\} \subset \Pi^{ \pm}, n=1,2, \ldots$ with $\theta_{s_{n}} \tilde{\phi}:=\left(\varphi\left(\delta_{+}\left(s_{n}, t\right)\right), \delta_{-}\left(s_{n}, T\right)\right)$, we shall consider the sets $\left\{\varphi\left(\delta_{+}\left(s_{n}, t\right)\right), \delta_{-}\left(s_{n}, T\right)\right\} \subset P C_{r d} \times \mathbb{B}$, where

$$
\delta_{-}\left(s_{n}, T\right)=\left\{\delta_{-}\left(s_{n}, t_{k}\right): k \in \mathbb{Z}, n=1,2, \ldots\right\} .
$$

Definition 5. The sequence $\left\{\tilde{\phi}_{n}\right\}, \tilde{\phi}_{n}=\left(\varphi_{n}(t), T_{n}\right) \in P C_{r d}(\mathbb{T}, \mathbb{X}) \times \mathbb{B}$ is convergent to $\tilde{\phi}^{*}$ pointwise, $\tilde{\phi}^{*}=\left(\varphi^{*}(t), T^{*}\right),\left(\varphi^{*}(t), T^{*}\right) \in P C_{r d}(\mathbb{T}, \mathbb{X}) \times \mathbb{B}$, if and only if for any $\varepsilon>0$ there exists $n_{0}>0$ such that $n \geq n_{0}$ implies

$$
d\left(T_{n}, T^{*}\right)<\varepsilon,\left\|\varphi_{n}(t)-\varphi^{*}(t)\right\|<\varepsilon \text { pointwise }
$$

for $t \in \mathbb{T} \backslash F_{\varepsilon}\left(s\left(T_{n} \cup T^{*}\right)\right), d(\cdot, \cdot)$ is an arbitrary distance in $\mathbb{B}$.

For convenience, consider the metric space $B P C_{r d}(\mathbb{T}, \mathbb{X}) \times \mathbb{B}$ with the metric

$$
d_{\infty}=\sup _{t \in \mathbb{T} \backslash \mathbb{B}} \tilde{d}\left(\tilde{\phi}_{t}, \tilde{\phi}_{t}^{*}\right),
$$

where

$$
\tilde{d}\left(\tilde{\phi}_{t}, \tilde{\phi}_{t}^{*}\right)=\max \left\{d\left(T, T^{*}\right),\left\|\varphi(t)-\varphi^{*}(t)\right\|\right\},
$$

and $\tilde{\phi}=(\varphi(t), T), \tilde{\phi}^{*}=\left(\varphi^{*}(t), T^{*}\right)$.

Theorem 1. The metric space $\left(B P C_{r d}(\mathbb{T}, \mathbb{X}) \times \mathbb{B}, d_{\infty}\right)$ is complete.

Proof. For any given Cauchy sequence $\left\{\tilde{\phi}_{n}=\left(\varphi_{n}(t), T_{n}\right)\right\} \subset B P C_{r d}(\mathbb{T}, \mathbb{X}) \times \mathbb{B}$, we can obtain that the sequences $\left\{\varphi_{n}(t)\right\}$ and $\left\{T_{n}\right\}$ be the Cauchy sequences in the metric space $\left(B P C_{r d}(\mathbb{T}, \mathbb{X}),\|\cdot\|_{\infty}\right)$ and $(\mathbb{B}, d)$ respectively. Hence, for any $\varepsilon>0$, there exists some $n_{0}>0$ such that $n, m>n_{0}$ implies $d\left(T_{n}, T_{m}\right)<\varepsilon$, which yields that $\left|t_{k}^{(n)}-t_{k}^{(m)}\right|<\varepsilon$ for any $k \in \mathbb{Z}$. Thus there exists $t_{k}$ such that $n>n_{0}$ 
implies $\left|t_{k}^{(n)}-t_{k}\right|<\varepsilon$. Therefore, there exists $\left\{t_{k}\right\}=T$ such that $n>n_{0}$ implies $d\left(T_{n}, T\right)<\varepsilon$. Moreover, for $n>n_{0}$, we can obtain

$$
\begin{aligned}
\delta_{-}\left(t_{k}, t_{k+1}\right) & =\left(\left|\delta_{+}\left(t_{k+1}, \delta_{-}\left(t_{k+1}^{(n)}, \delta_{-}\left(t_{k}, \delta_{+}\left(t_{k}^{(n)}, \delta_{-}\left(t_{k}^{(n)}, t_{k+1}^{(n)}\right)\right)\right)\right)\right)\right|\right. \\
& =\left|\delta_{+}\left(t_{k+1}, \delta_{-}\left(t_{k+1}^{(n)}, \delta_{+}\left(\delta_{-}\left(t_{k}, t_{k}^{(n)}\right), \delta_{-}\left(t_{k}^{(n)}, t_{k+1}^{(n)}\right)\right)\right)\right)\right| \\
& =\left|\delta_{-}\left(\delta_{-}\left(t_{k+1}, t_{k+1}^{(n)}\right), \delta_{+}\left(\delta_{-}\left(t_{k}, t_{k}^{(n)}\right), \delta_{-}\left(t_{k}^{(n)}, t_{k+1}^{(n)}\right)\right)\right)\right| \\
& =\left|\delta_{+}\left(\delta_{-}\left(\delta_{-}\left(t_{k+1}, t_{k+1}^{(n)}\right), \delta_{-}\left(t_{k}, t_{k}^{(n)}\right)\right), \delta_{-}\left(t_{k}^{(n)}, t_{k+1}^{(n)}\right)\right)\right| \\
& \geq\left|\delta_{-}\left(A\left(\delta_{-}\left(\delta_{-}\left(t_{k+1}, t_{k+1}^{(n)}\right), \delta_{-}\left(t_{k}, t_{k}^{(n)}\right)\right)\right), \delta_{-}\left(t_{k}^{(n)}, t_{k+1}^{(n)}\right)\right)\right| \\
& =\left|\delta_{-}\left(A\left(t_{0}\right)+o(\alpha), \delta_{-}\left(t_{k}^{(n)}, t_{k+1}^{(n)}\right)\right)\right| \geq\left|\delta_{-}\left(A\left(t_{0}\right)+o(\alpha), \theta_{n}\right)\right|,
\end{aligned}
$$

where $\theta_{n}=\inf _{k \in \mathbb{Z}} \delta_{-}\left(t_{k}^{(n)}, t_{k+1}^{(n)}\right)>0$ and $\lim _{n \rightarrow \infty} o(\alpha)=0$, thus there exists some $n_{0}>0$ such that $n>n_{0}$ implies

$$
\inf _{k \in \mathbb{Z}} \delta_{-}\left(t_{k}, t_{k+1}\right) \geq\left|\delta_{-}\left(A\left(t_{0}\right)+o(\alpha), \theta_{n}\right)\right|>0,
$$

so we obtain $T \in \mathbb{B}$, which indicates $(\mathbb{B}, d)$ is complete.

For any fixed $t \in \mathbb{T} \backslash F_{\mathcal{E}}\left(s\left(T_{n} \cup T\right)\right), \varphi_{n}(t) \subset B P C_{r d}(\mathbb{T}, \mathbb{X})$ is a Cauchy sequence in the Banach space $\mathbb{X}$, hence for any $\varepsilon>0$, there exists some $\varphi(t) \in \mathbb{X}$ such that $n>n_{0}$ implies

$$
\left\|\varphi_{n}(t)-\varphi(t)\right\|<\varepsilon \text { pointwise. }
$$

We claim that $\varphi(t)$ is also bounded on $\mathbb{T} \backslash F_{\varepsilon}\left(s\left(T_{n} \cup T\right)\right)$. In fact, there exists some $n_{0}>0$ such that $n, m>n_{0}$ implies $\left\|\varphi_{n}-\varphi_{m}\right\|_{\infty}<1$, so for all $t \in \mathbb{T} \backslash F_{\varepsilon}\left(s\left(T_{n} \cup T\right)\right)$ we have

$$
|\varphi(t)|<\left|\varphi(t)-\varphi_{n_{0}}(t)\right|+\left|\varphi_{n_{0}}(t)\right|<\left\|\varphi-\varphi_{n_{0}}\right\|_{\infty}+\left\|\varphi_{n_{0}}\right\|_{\infty} \leq 1+\left\|\varphi_{n_{0}}\right\|_{\infty} .
$$

To complete the proof, it is sufficient to show $\varphi_{n} \rightarrow \varphi$ in norm on $\mathbb{T} \backslash F_{\mathcal{E}}\left(s\left(T_{n} \cup T\right)\right)$, i.e., $\| \varphi-$ $\varphi_{n} \| \rightarrow 0$ as $n \rightarrow \infty$. According to (5), there exists some $n_{0}>0$ such that $n, m>n_{0}$ implies

$$
\begin{aligned}
\left\|\varphi-\varphi_{n}\right\|_{\infty} & \leq\left\|\varphi_{n}-\varphi_{n_{0}}\right\|_{\infty}+\left\|\varphi-\varphi_{n_{0}}\right\|_{\infty} \\
& \leq\left\|\varphi_{n}-\varphi_{n_{0}}\right\|_{\infty}+\lim _{m \rightarrow \infty}\left\|\varphi_{m}-\varphi_{n_{0}}\right\|_{\infty} \leq \varepsilon+\varepsilon=2 \varepsilon
\end{aligned}
$$

for all $t \in \mathbb{T} \backslash F_{\varepsilon}\left(s\left(T_{n} \cup T\right)\right)$, so $\left\|\varphi-\varphi_{n}\right\|_{\infty} \leq \varepsilon$. This completes the proof.

Definition 6. Let $\mathbb{T}$ be a bi-direction S-CCTS. A function $\varphi \in B P C_{r d}(\mathbb{T}, \mathbb{X})$ is said to be rd-piecewise S-almost automorphic if the following conditions are fulfilled:

(i) Let $T=\left\{t_{k}\right\}$ be a S-equipotentially almost automorphic sequence.

(ii) Let $\varphi \in B P C_{r d}(\mathbb{T}, \mathbb{X})$ be a bounded function with respect to a sequence $T=\left\{t_{k}\right\}$. Then $\varphi$ is said to be piecewise S-almost automorphic if from every sequence $\left\{s_{n}\right\}_{n=1}^{\infty} \subset \Pi^{ \pm}$, we can extract a subsequence $\left\{\tau_{n}\right\}_{n=1}^{\infty}$ such that

$$
\tilde{\phi}^{*}=\left(\varphi^{*}(t), T^{*}\right)=\lim _{n \rightarrow \infty}\left(\varphi\left(\delta_{+}\left(\tau_{n}, t\right)\right), \delta_{-}\left(\tau_{n}, T\right)\right)=\lim _{n \rightarrow \infty} \theta_{\tau_{n}} \tilde{\phi}
$$

is well defined for each $t \in \mathbb{T}$ and

$$
\tilde{\phi}=(\varphi(t), T)=\lim _{n \rightarrow \infty}\left(\varphi^{*}\left(\delta_{-}\left(\tau_{n}, t\right)\right), \delta_{+}\left(\tau_{n}, T^{*}\right)\right)=\lim _{n \rightarrow \infty} \theta_{-} \tau_{n} \tilde{\phi}^{*}
$$

for each $t \in \mathbb{T}$. Denote by $A A_{S}(\mathbb{T}, \mathbb{X})$ the set of all such functions. 
(iii) A bounded function $f \in B P C_{r d}(\mathbb{T} \times \mathbb{X}, \mathbb{X})$ with respect to a sequence $T=\left\{t_{k}\right\}$ is said to be piecewise $S$-almost automorphic if $f(t, x)$ is piecewise S-automorphic in $t \in \mathbb{T}$ uniformly for $x \in B$, where $B$ is any bounded subset of $\mathbb{X}$. Denote by $A A_{S}(\mathbb{T} \times \mathbb{X}, \mathbb{X})$ the set of all such functions.

Similarly, we can also introduce the concept of piecewise $S$-almost automorphic functions that belong to $P C_{r d}(\mathbb{T}, \mathbb{X})$.

Let $U$ be the set of all functions $\rho: \mathbb{T} \rightarrow(0, \infty)$ which are positive and locally $\Delta$-integrable over $\mathbb{T}$. For a given $r \in(0, \infty)_{\Pi^{ \pm}}$and $\forall t_{0} \in \mathbb{T}^{*}$, set

$$
m_{S}\left(r, \rho, t_{0}\right):=\int_{\delta_{-}\left(r, t_{0}\right)}^{\delta_{+}\left(r, t_{0}\right)} \rho(s) \Delta s
$$

for each $\rho \in U$.

Remark 7. Particularly, if we let $\mathbb{T}=\left\{q^{n}: n \in \mathbb{Z}, q>1\right\} \cup\{0\}$ and $t_{0}=q$, then (6) will turn into the integral on the quantum time scale:

$$
m_{S}\left(r, \rho, t_{0}\right):=\int_{\frac{q}{r}}^{q r} \rho(s) \Delta s .
$$

Moreover, let $\mathbb{T}=\{ \pm \sqrt{n}: n \in \mathbb{N}\}$ and $t_{0}=1$, then the integral is

$$
m_{S}\left(r, \rho, t_{0}\right):=\int_{-\sqrt{1+r^{2}}}^{\sqrt{1+r^{2}}} \rho(s) \Delta s .
$$

Define

$$
\begin{gathered}
U_{\infty}:=\left\{\rho \in U: \lim _{r \rightarrow \infty} m_{S}\left(r, \rho, t_{0}\right)=\infty\right\}, \\
U_{B}:=\left\{\rho \in U_{\infty}: \rho \text { is bounded and } \inf _{s \in \mathbb{T}} \rho(s)>0\right\} .
\end{gathered}
$$

It is clear that $U_{B} \subset U_{\infty} \subset U$. Now for $\rho \in U_{\infty}$ define

$$
\begin{aligned}
& \operatorname{WPAA}_{0}^{S}(\mathbb{T}, \rho):=\left\{\phi \in \operatorname{BPC}_{r d}(\mathbb{T}, \mathbb{X}):\right. \\
& \left.\lim _{r \rightarrow \infty} \frac{1}{m_{S}\left(r, \rho, t_{0}\right)} \int_{\delta_{-}\left(r, t_{0}\right)}^{\delta_{+}\left(r, t_{0}\right)}\|\phi(s)\| \rho(s) \Delta s=0, \forall t_{0} \in \mathbb{T}, r \in \Pi^{ \pm}\right\} .
\end{aligned}
$$

Similarly, we define

$$
\begin{aligned}
& W P A A_{0}^{S}(\mathbb{T} \times \mathbb{X}, \rho): \\
= & \left\{\Phi \in B P C_{r d}(\mathbb{T} \times \Omega, \mathbb{X}): \lim _{r \rightarrow \infty} \frac{1}{m_{S}\left(r, \rho, t_{0}\right)} \int_{\delta_{-}\left(r, t_{0}\right)}^{\delta_{+}\left(r, t_{0}\right)}\|\Phi(s, x)\| \rho(s) \Delta s=0\right. \\
& \text { uniformly with respect to } \left.x \in K, \forall t_{0} \in \mathbb{T}, r \in \Pi^{ \pm}\right\} .
\end{aligned}
$$

We are now ready to introduce the sets $W P A A_{S}(\mathbb{T}, \rho)$ and $W P A A_{S}(\mathbb{T} \times \mathbb{X}, \rho)$ of weighted pseudo $S$-almost automorphic functions:

$$
\begin{aligned}
& W P A A_{S}(\mathbb{T}, \rho)=\left\{f=g+\phi \in B P C_{r d}(\mathbb{T}, \mathbb{X}): g \in A A_{S}(\mathbb{T}, \mathbb{X}) \text { and } \phi \in W P A A_{0}^{S}(\mathbb{T}, \rho)\right\}, \\
& W P A A_{S}(\mathbb{T} \times \mathbb{X}, \rho)=\left\{f=g+\phi \in B P C_{r d}(\mathbb{T} \times \mathbb{X}, \mathbb{X}): g \in A A_{S}(\mathbb{T} \times \mathbb{X}, \mathbb{X})\right. \\
& \text { and } \left.\phi \in W P A A_{0}^{S}(\mathbb{T} \times \mathbb{X}, \rho)\right\} .
\end{aligned}
$$


Lemma 3. Let $\mathbb{T}$ be bi-direction S-CCTS under shifts $\delta_{ \pm}$and $\phi \in B P C_{r d}(\mathbb{T}, \mathbb{X})$. Then $\phi \in W P A A_{0}^{S}(\mathbb{T}, \rho)$ where $\rho \in U_{B}$ if and only if for every $\varepsilon>0$,

$$
\lim _{r \rightarrow \infty} \frac{1}{m_{S}\left(r, \rho, t_{0}\right)} \mu_{\Delta}\left(M_{r, \varepsilon, t_{0}}(\phi)\right)=0,
$$

where $r \in \Pi^{ \pm}, \mu_{\Delta}(\cdot)$ is the $\Delta$-measurability function on the time scale $\mathbb{T}$ and

$$
M_{r, \varepsilon, t_{0}}(\phi):=\left\{t \in\left[\delta_{-}\left(r, t_{0}\right), \delta_{+}\left(r, t_{0}\right)\right]_{\mathbb{T}^{*}}:\|\phi(t)\| \geq \varepsilon\right\} .
$$

\section{Proof.}

(a) Necessity. For contradiction, suppose that there exists $\varepsilon_{0}>0$ such that

$$
\lim _{r \rightarrow \infty} \frac{1}{m_{S}\left(r, \rho, t_{0}\right)} \mu_{\Delta}\left(M_{r, \varepsilon_{0}, t_{0}}(\phi)\right) \neq 0 .
$$

Then there exists $\delta>0$ such that for every $n \in \mathbb{N}$,

$$
\frac{1}{m_{S}\left(r_{n}, \rho, t_{0}\right)} \mu_{\Delta}\left(M_{r_{n}, \varepsilon_{0}, t_{0}}(\phi)\right) \geq \delta \text { for some } r_{n}>n, \text { where } r_{n} \in \Pi^{ \pm} .
$$

So we get

$$
\begin{aligned}
& \frac{1}{m_{S}\left(r_{n}, \rho, t_{0}\right)} \int_{\delta_{-}\left(r, t_{0}\right)}^{\delta_{+}\left(r, t_{0}\right)}\|\phi(s)\| \rho(s) \Delta s \\
= & \frac{1}{m_{S}\left(r_{n}, \rho, t_{0}\right)} \int_{M_{r_{n}, \varepsilon_{0}, t_{0}}(\phi)}\|\phi(s)\| \rho(s) \Delta s+\frac{1}{m_{S}\left(r_{n}, \rho, t_{0}\right)} \\
& \times \int_{\left[\delta_{-}\left(r, t_{0}\right), \delta_{+}\left(r, t_{0}\right)\right]_{\mathbb{T}^{*}} \backslash M_{r_{n}, \varepsilon_{0}, t_{0}}(\phi)}\|\phi(s)\| \rho(s) \Delta s \\
\geq & \frac{1}{m_{S}\left(r_{n}, \rho, t_{0}\right)} \int_{M_{r_{n}, \varepsilon_{0}, t_{0}}(\phi)}\|\phi(s)\| \rho(s) \Delta s \\
\geq & \frac{\varepsilon_{0}}{m_{S}\left(r_{n}, \rho, t_{0}\right)} \int_{M_{r_{n}, \varepsilon_{0}, t_{0}}(\phi)}\|\phi(s)\| \rho(s) \Delta s \geq \varepsilon_{0} \delta \gamma,
\end{aligned}
$$

where $\gamma=\inf _{s \in \mathbb{T}} \rho(s)$. This contradicts the assumption.

(b) Sufficiency. Assume that $\lim _{r \rightarrow \infty} \frac{1}{m_{S}\left(r, \rho, t_{0}\right)} \mu_{\Delta}\left(M_{r, \varepsilon, t_{0}}(\phi)\right)=0$. Then for every $\varepsilon>0$, there exists $r_{0}>0$ such that for every $r>r_{0}, \frac{1}{m_{S}\left(r, \rho, t_{0}\right)} \mu_{\Delta}\left(M_{r, \varepsilon, t_{0}}(\phi)\right)<\frac{\varepsilon}{K M}$, where $M:=\sup _{t \in \mathbb{T}}\|\phi(t)\|<\infty$ and $K:=\sup _{t \in \mathbb{T}} \rho(t)<\infty$.

Now, we have

$$
\begin{aligned}
& \frac{1}{m_{S}\left(r, \rho, t_{0}\right)} \int_{\delta_{-}\left(r, t_{0}\right)}^{\delta_{+}\left(r, t_{0}\right)}\|\phi(s)\| \rho(s) \Delta s \\
= & \frac{1}{m_{S}\left(r, \rho, t_{0}\right)}\left(\int_{M_{r, \varepsilon, t_{0}}(\phi)}\|\phi(s)\| \rho(s) \Delta s\right. \\
& \left.+\int_{\left[\delta_{-}\left(r, t_{0}\right), \delta_{+}\left(r, t_{0}\right)\right]_{\mathbb{T}^{*}} \backslash M_{r, \varepsilon, t_{0}}(\phi)}\|\phi(s)\| \rho(s) \Delta s\right) \\
\leq & \frac{M K}{m_{S}\left(r, \rho, t_{0}\right)} \mu_{\Delta}\left(M_{r, \varepsilon, t_{0}}(\phi)\right) \\
& +\frac{\varepsilon}{m_{S}\left(r, \rho, t_{0}\right)} \int_{\left[\delta_{-}\left(r, t_{0}\right), \delta_{+}\left(r, t_{0}\right)\right]_{\mathbb{T}^{*} \backslash M_{r, \varepsilon, t_{0}}(\phi)} \rho(s) \Delta s \leq 2 \varepsilon .}
\end{aligned}
$$


Therefore, $\lim _{r \rightarrow \infty} \frac{1}{m_{S}\left(r, \rho, t_{0}\right)} \int_{\delta_{-}\left(r, t_{0}\right)}^{\delta_{+}\left(r, t_{0}\right)}\|\phi(s)\| \rho(s) \Delta s=0$, that is, $\phi \in W P A A_{0}^{S}(\mathbb{T}, \rho)$. This completes the proof.

Lemma 4. Let $\mathbb{T}$ be bi-direction S-CCTS under shifts $\delta_{ \pm}$. Then WPA $A_{0}^{S}(\mathbb{T}, \rho)$ is a shift invariant set of $B P C_{r d}(\mathbb{T}, \mathbb{X})$ with respect to $\Pi^{ \pm}$if $\rho \in U_{B}$, i.e., for any $s \in \Pi^{ \pm}$, one has $\phi\left(\delta_{+}(s, t)\right):=\theta_{s} \phi \in W P A A_{0}^{S}(\mathbb{T}, \rho)$ if $\rho \in U_{B}$.

Proof. For any $s \in \Pi^{ \pm}, \phi \in W P A A_{0}^{S}(\mathbb{T}, \rho), \varepsilon>0, r>0$, we have

$$
\begin{aligned}
M_{r, \varepsilon, t_{0}}\left(\theta_{s} \phi\right) & =\left\{t \in\left[\delta_{-}\left(r, t_{0}\right), \delta_{+}\left(r, t_{0}\right)\right]_{\mathbb{T}^{*}}:\left\|\theta_{\mathcal{S}}(t)\right\| \geq \varepsilon\right\} \\
& =\left\{t \in\left[\delta_{-}\left(r, t_{0}\right), \delta_{+}\left(r, t_{0}\right)\right]_{\mathbb{T}^{*}}:\left\|\phi\left(\delta_{+}(s, t)\right)\right\| \geq \varepsilon\right\} \\
& =\left\{t \in\left[\delta_{+}\left(s, \delta_{-}\left(r, t_{0}\right)\right), \delta_{+}\left(s, \delta_{+}\left(r, t_{0}\right)\right)\right]_{\mathbb{T}^{*}}:\|\phi(t)\| \geq \varepsilon\right\} \\
& \subseteq\left\{t \in\left[\delta_{-}\left(\delta_{-}(s, r), t_{0}\right), \delta_{+}\left(\delta_{+}(s, r), t_{0}\right)\right]_{\mathbb{T}^{*}}:\|\phi(t)\| \geq \varepsilon\right\} \\
& \subseteq\left\{t \in\left[\delta_{-}\left(\delta_{-}(A(s), r), t_{0}\right), \delta_{+}\left(\delta_{+}(A(s), r), t_{0}\right)\right]_{\mathbb{T}^{*}}:\|\phi(t)\| \geq \varepsilon\right\} \\
& \subseteq\left\{t \in\left[\delta_{-}\left(\delta_{+}(A(s), r), t_{0}\right), \delta_{+}\left(\delta_{+}(A(s), r), t_{0}\right)\right]_{\mathbb{T}^{*}}:\|\phi(t)\| \geq \varepsilon\right\}
\end{aligned}
$$

Hence

$$
\begin{aligned}
& \frac{1}{m_{S}\left(r, \rho, t_{0}\right)} \mu_{\Delta}\left(M_{r, \varepsilon, t_{0}}\left(\theta_{S} \phi\right)\right) \leq \frac{1}{m_{S}\left(r, \rho, t_{0}\right)} \mu_{\Delta}\left(M_{\delta_{+}}(A(s), r), \varepsilon, t_{0}\left(\theta_{S} \phi\right)\right) \\
= & \frac{m_{S}\left(\delta_{+}(A(s), r), \rho, t_{0}\right)}{m_{S}\left(r, \rho, t_{0}\right)} \frac{1}{m_{S}\left(\delta_{+}(A(s), r), \rho, t_{0}\right)} \mu_{\Delta}\left(M_{\delta_{+}(A(s), r), \varepsilon, t_{0}}(\phi)\right) .
\end{aligned}
$$

Since $\phi \in W P A A_{0}^{S}(\mathbb{T}, \rho)$, then by Lemma 3, we have

$$
\frac{1}{m_{S}\left(\delta_{+}(A(s), r), \rho, t_{0}\right)}\left(M_{\delta_{+}}(A(s), r), \varepsilon, t_{0}(\phi)\right) \rightarrow 0 \text { as } r \rightarrow \infty .
$$

Furthermore, $\lim _{r \rightarrow \infty} \frac{m_{S}\left(\delta_{+}(A(s), r), \rho, t_{0}\right)}{m_{S}\left(r, p, t_{0}\right)}=1$, thus

$$
\frac{1}{m_{S}\left(r, \rho, t_{0}\right)} \mu_{\Delta}\left(M_{r, \varepsilon, t_{0}}\left(\theta_{S}(\phi)\right)\right) \rightarrow 0, r \rightarrow \infty
$$

Again, using Lemma 3, one can get $\theta_{s} \phi \in W P A A_{0}^{S}(\mathbb{T}, \rho)$ for any $s \in \Pi^{ \pm}$. This completes the proof.

By Definition 6, the following two Lemmas are obvious.

Lemma 5. Let $\mathbb{T}$ be bi-direction S-CCTS under shifts $\delta_{ \pm}$and $\phi \in A A_{S}(\mathbb{T}, \mathbb{X})$, then the range of $\phi, \phi(\mathbb{T})$, is a relatively compact subset of $\mathbb{X}$.

Lemma 6. Let $\mathbb{T}$ be bi-direction S-CCTS under shifts $\delta_{ \pm}$. If $f=g+\phi$ with $g \in A A_{S}(\mathbb{T}, \mathbb{X})$, and $\phi \in$ $W P A A_{0}^{S}(\mathbb{T}, \rho)$, where $\rho \in U_{B}$, then $g(\mathbb{T}) \subset \overline{f(\mathbb{T})}$.

Lemma 7. Let $\mathbb{T}$ be bi-direction S-CCTS under shifts $\delta_{ \pm}$. The decomposition of a weighted piecewise pseudo S-almost automorphic function according to $A A_{S} \oplus W P A A_{0}^{S}$ is unique for any $\rho \in U_{B}$. 
Proof. Let $f \in W P A A_{S}(\mathbb{T}, \rho)$, if $f=g_{1}+\phi_{1}=g_{2}+\phi_{2}$, then we have $g_{1}-g_{2}=\phi_{2}-\phi_{1}$. Hence, we obtain that there exists some positive constant $c$ such that

$$
\begin{aligned}
c \cdot \sup _{t \in \mathbb{T}}\left\|g_{1}(t)-g_{2}(t)\right\| \leq & \lim _{r \rightarrow \infty} \frac{1}{m_{S}\left(r, \rho, t_{0}\right)} \int_{\delta_{-}\left(r, t_{0}\right)}^{\delta_{+}\left(r, t_{0}\right)}\left\|g_{1}(s)-g_{2}(s)\right\| \rho(s) \Delta s \\
= & \lim _{r \rightarrow \infty} \frac{1}{m_{S}\left(r, \rho, t_{0}\right)} \int_{\delta_{-}\left(r, t_{0}\right)}^{\delta_{+}\left(r, t_{0}\right)}\left\|\phi_{1}(s)-\phi_{2}(s)\right\| \rho(s) \Delta s \\
\leq & \lim _{r \rightarrow \infty} \frac{1}{m_{S}\left(r, \rho, t_{0}\right)} \int_{\delta_{-}\left(r, t_{0}\right)}^{\delta_{+}\left(r, t_{0}\right)}\left\|\phi_{1}(s)\right\| \rho(s) \Delta s \\
& +\lim _{r \rightarrow \infty} \frac{1}{m_{S}\left(r, \rho, t_{0}\right)} \int_{\delta_{-}\left(r, t_{0}\right)}^{\delta_{+}\left(r, t_{0}\right)}\left\|\phi_{2}(s)\right\| \rho(s) \Delta s=0,
\end{aligned}
$$

so $g_{1}-g_{2}=0=\phi_{1}-\phi_{2}$, i.e., $g_{1}=g_{2}$ and $\phi_{1}=\phi_{2}$. This completes the proof.

Lemma 8. Let $\left\{\varphi_{n}\right\} \subset A A_{S}(\mathbb{T}, \mathbb{X})$ be a sequence of piecewise S-almost automorphic functions such that $\lim _{n \rightarrow \infty} \varphi_{n}=\varphi$ uniformly, then $\varphi$ is piecewise S-almost automorphic.

Proof. From Definition 6, denote $\tilde{\phi}_{n}=\left(\varphi_{n}(t), T_{n}\right)$ and $\tilde{\phi}^{*}=(\varphi(t), T)$. Let $\left\{\tau_{n}^{\prime}\right\}$ be an arbitrary sequence of real numbers. Then we can extract a subsequence $\left\{\tau_{n}\right\}$ of $\left\{\tau_{n}^{\prime}\right\} \subset \Pi^{ \pm}$such that

$$
\lim _{n \rightarrow \infty} \theta_{\tau_{n}} \tilde{\phi}_{i}=\tilde{\phi}_{i}^{*}
$$

for each $i=1,2, \ldots$, pointwise.

We claim that the sequence of functions $\left\{\tilde{\phi}_{i}^{*}\right\}$ is a Cauchy sequence. In fact, we can obtain

$$
\tilde{d}\left(\tilde{\phi}_{i}^{*}, \tilde{\phi}_{j}^{*}\right) \leq \tilde{d}\left(\tilde{\phi}_{i}^{*}, \theta_{\tau_{n}} \tilde{\phi}_{i}\right)+\tilde{d}\left(\theta_{\tau_{n}} \tilde{\phi}_{i}, \theta_{\tau_{n}} \tilde{\phi}_{j}\right)+\tilde{d}\left(\theta_{\tau_{n}} \tilde{\phi}_{j}, \tilde{\phi}_{j}^{*}\right) .
$$

Let $\varepsilon>0$. By the uniform convergence of $\left\{\tilde{\phi}_{n}\right\}$ there exists a positive integer $N$ such that for all $i, j>N$ implies $\tilde{d}\left(\theta_{\tau_{n}} \tilde{\phi}_{i}, \theta_{\tau_{n}} \tilde{\phi}_{j}\right)<\varepsilon$. By using (7), (8) and the completeness of the space $\mathbb{X}$, we can deduce the pointwise convergence of the sequence $\left\{\tilde{\phi}_{n}\right\}$, say to a function $\tilde{\phi}$.

Now, we claim that $\lim _{n \rightarrow \infty} \tilde{\phi}_{\tau_{n}}=\tilde{\phi}^{*}$ and $\lim _{n \rightarrow \infty} \tilde{\phi}_{-\tau_{n}}^{*}=\tilde{\phi}$ pointwise on $\mathbb{T}$.

Let $\varepsilon>0$, there exists some positive integer $M$ such that $\tilde{d}\left(\theta_{\tau_{n}} \tilde{\phi}, \theta_{\tau_{n}} \tilde{\phi}_{M}\right)<\varepsilon$ and $\tilde{d}\left(\tilde{\phi}_{M}^{*}, \tilde{\phi}^{*}\right)<\varepsilon$ pointwise so that

$$
\tilde{d}\left(\tilde{\phi}_{\tau_{n}}, \tilde{\phi}^{*}\right) \leq 2 \varepsilon+\tilde{d}\left(\theta_{\tau_{n}} \tilde{\phi}_{M}, \tilde{\phi}_{M}^{*}\right) \text { pointwise, }
$$

since for each $M$, there exists some positive integer $K=K(t, M)$ such that $\tilde{d}\left(\theta_{\tau_{n}} \tilde{\phi}_{M}, \tilde{\phi}_{M}^{*}\right)<\varepsilon$, then we can obtain $\tilde{d}\left(\tilde{\phi}_{\tau_{n}}, \tilde{\phi}^{*}\right) \leq 3 \varepsilon$ for $n \geq N_{0}$, where $N_{0}$ is some positive integer depending on $t$ and $\varepsilon$.

Similarly, the same step can be applied for $\lim _{n \rightarrow \infty} \tilde{\phi}_{-\tau_{n}}^{*}=\tilde{\phi}$ pointwise on $\mathbb{T}$, thus, we can obtain the desired result. This completes the proof.

Theorem 2. Let $\mathbb{T}$ be bi-direction S-CCTS under shifts $\delta_{ \pm}$. For $\rho \in U_{B},\left(W P A A_{S}(\mathbb{T} \times \mathbb{X}, \rho),\|\cdot\|_{\infty}\right)$ is a Banach space.

Proof. For any convergent sequence $\left\{f_{n}\right\} \subset W P A A_{0}^{S}(\mathbb{T} \times \mathbb{X}, \rho)$ with $f_{n} \rightarrow f$ uniformly for $t \in \mathbb{T}$, we can obtain

$$
\begin{aligned}
& \lim _{r \rightarrow \infty} \frac{1}{m_{S}\left(r, \rho, t_{0}\right)} \int_{\delta_{-}\left(r, t_{0}\right)}^{\delta_{+}\left(r, t_{0}\right)}\|f(s, x)\| \rho(s) \Delta s \\
\leq \quad & \lim _{r \rightarrow \infty} \frac{1}{m_{S}\left(r, \rho, t_{0}\right)} \int_{\delta_{-}\left(r, t_{0}\right)}^{\delta_{+}(r)}\left\|f_{n}(s, x)\right\| \rho(s) \Delta s \\
& +\lim _{r \rightarrow \infty} \frac{1}{m_{S}\left(r, \rho, t_{0}\right)} \int_{\delta_{-}\left(r, t_{0}\right)}^{\delta_{+}\left(r, t_{0}\right)}\left\|f_{n}(s, x)-f(s, x)\right\| \rho(s) \Delta s,
\end{aligned}
$$


by letting $n \rightarrow \infty$ we have

$$
\lim _{r \rightarrow \infty} \frac{1}{m_{S}\left(r, \rho, t_{0}\right)} \int_{\delta_{-}\left(r, t_{0}\right)}^{\delta_{+}\left(r, t_{0}\right)}\|f(s, x)\| \rho(s) \Delta s=0
$$

which indicates $W P A A_{0}^{S}(\mathbb{T} \times \mathbb{X}, \rho)$ is a closed subspace of $B P C_{r d}(\mathbb{T} \times \Omega, \mathbb{X})$. Therefore, $W P A A_{0}^{S}(\mathbb{T} \times$ $\mathbb{X}, \rho)$ is itself a Banach space. Then by Lemmas 7 and 8 , we have $\left(W P A A_{S}(\mathbb{T} \times \mathbb{X}, \rho),\|\cdot\|_{\infty}\right)$ is a Banach space. The proof is completed.

Definition 7. Let $\rho_{1}, \rho_{2} \in U_{\infty}$. One says that $\rho_{1}$ equivalent $\rho_{2}$, written $\rho_{1} \sim \rho_{2}$ if $\rho_{1} / \rho_{2} \in U_{B}$.

Theorem 3. Let $\rho_{1}, \rho_{2} \in U_{\infty}$. If $\rho_{1} \sim \rho_{2}$, then $W P A A_{S}\left(\mathbb{T}, \rho_{1}\right)=W P A A_{S}\left(\mathbb{T}, \rho_{2}\right)$.

Proof. Assume that $\rho_{1} \sim \rho_{2}$. There exist $a, b>0$ such that $a \rho_{1} \leq \rho_{2} \leq b \rho_{1}$. So

$$
a m_{S}\left(r, \rho_{1}, t_{0}\right) \leq m_{S}\left(r, \rho_{2}, t_{0}\right) \leq b m_{S}\left(r, \rho_{1}, t_{0}\right),
$$

where $r \in \Pi^{ \pm}$, and

$$
\begin{aligned}
\frac{a}{b} \frac{1}{m_{S}\left(r, \rho_{1}, t_{0}\right)} \int_{\delta_{-}\left(r, t_{0}\right)}^{\delta_{+}\left(r, t_{0}\right)}\|\phi(s)\| \rho_{1}(s) \Delta s & \leq \frac{1}{m_{S}\left(r, \rho_{2}, t_{0}\right)} \int_{\delta_{-}\left(r, t_{0}\right)}^{\delta_{+}\left(r, t_{0}\right)}\|\phi(s)\| \rho_{2}(s) \Delta s \\
& \leq \frac{b}{a} \frac{1}{m_{S}\left(r, \rho_{1}, t_{0}\right)} \int_{\delta_{-}\left(r, t_{0}\right)}^{\delta_{+}\left(r, t_{0}\right)}\|\phi(s)\| \rho_{1}(s) \Delta s .
\end{aligned}
$$

This completes the proof.

Lemma 9. Let $\mathbb{T}$ be bi-direction $S$-CCTS under shifts $\delta_{ \pm}$. If $g \in A A_{S}(\mathbb{T} \times \mathbb{X}, \mathbb{X})$ and $\alpha \in A A_{S}(\mathbb{T}, \mathbb{X})$, then $G(t):=g(\cdot, \alpha(\cdot)) \in A A_{S}(\mathbb{T}, \mathbb{X})$.

Proof. Let $T=\left\{t_{k}\right\}, \tilde{\phi}=(g(t, x), T) \in A A_{S}(\mathbb{T} \times \mathbb{X}, \mathbb{X}) \times \mathbb{B}$, from every sequence $\left\{s_{n}\right\}_{n=1}^{\infty} \subset \Pi^{ \pm}$, we can extract a subsequence $\left\{\tau_{n}\right\}_{n=1}^{\infty}$ such that

$$
\tilde{\phi}^{*}:=\left(g^{*}(t, x), T^{*}\right)=\lim _{n \rightarrow \infty} \theta_{\tau_{n}} \tilde{\phi}=\lim _{n \rightarrow \infty}\left(g\left(\delta_{+}\left(\tau_{n}, t\right), x\right), \delta_{-}\left(\tau_{n}, T\right)\right),
$$

uniformly exists on $P C_{r d}(\mathbb{T} \times \mathbb{X}, \mathbb{X}) \times \mathbb{B}$. Since $\alpha \in A A_{S}(\mathbb{T}, \mathbb{X})$, one can extract $\left\{\tau_{n}^{\prime}\right\} \subset\left\{\tau_{n}\right\}$ such that

$$
\begin{aligned}
\lim _{n \rightarrow \infty} \theta_{\tau_{n}^{\prime}} \tilde{\phi} & =\lim _{n \rightarrow \infty}\left(g\left(\delta_{+}\left(\tau_{n}^{\prime}, t\right), \alpha\left(\delta_{+}\left(\tau_{n}^{\prime}, t\right)\right)\right), \delta_{-}\left(\tau_{n}^{\prime}, T\right)\right) \\
& =\lim _{n \rightarrow \infty}\left(g\left(\delta_{+}\left(\tau_{n}^{\prime}, t\right), \alpha^{*}(t)\right), \delta_{-}\left(\tau_{n}^{\prime}, T\right)\right)=\left(g^{*}\left(t, \alpha^{*}(t)\right), T^{*}\right) .
\end{aligned}
$$

Hence, $G \in A A_{S}(\mathbb{T}, \mathbb{X})$. This completes the proof.

By Lemmas 3 and 9, one can get the following theorem immediately without proof.

Theorem 4. Let $\mathbb{T}$ be bi-direction S-CCTS under shifts $\delta_{ \pm}$and $f=g+\phi \in W P A A_{S}(\mathbb{T} \times \mathbb{X}, \rho)$, where $g \in A A_{S}(\mathbb{T} \times \mathbb{X}, \mathbb{X}), \phi \in W P A A_{0}^{S}(\mathbb{T} \times \mathbb{X}, \rho), \rho \in U_{B}$ and the following conditions hold:

(i) $\{f(t, x): t \in \mathbb{T}, x \in K\}$ is bounded for every bounded subset $K \subseteq \Omega$.

(ii) $f(t, \cdot), g(t, \cdot)$ are uniformly continuous in each bounded subset of $\Omega$ uniformly in $t \in \mathbb{T}$.

Then $f(\cdot, h(\cdot)) \in W P A A_{S}(\mathbb{T}, \rho)$ if $h \in W P A A_{S}(\mathbb{T}, \rho)$ and $h(\mathbb{T}) \subset \Omega$.

Theorem 4 has the following consequence: 
Corollary 1. Let $f=g+\phi \in W P A A_{S}(\mathbb{T}, \rho)$, where $\rho \in U_{B}$. Assume that $f$ and $g$ are Lipschitzian in $x \in \mathbb{X}$ uniformly in $t \in \mathbb{T}$. Then $f(\cdot, h(\cdot)) \in W P A A_{S}(\mathbb{T}, \rho)$ if $h \in W P A A_{S}(\mathbb{T}, \rho)$.

Next, we will show the following two Lemmas, which are useful in the proof of our results.

Lemma 10. Let $\mathbb{T}$ be bi-direction S-CCTS under shifts $\delta_{ \pm}$. If $\varphi \in P C_{r d}(\mathbb{T}, \mathbb{X})$ is an S-almost automorphic function with respect to the sequence $T$ and $\left\{t_{k}\right\} \subset \mathbb{T}$ is S-equipotentially almost automorphic satisfying $\inf _{k \in \mathbb{Z}} t_{k}^{q}=\theta>t_{0}, q \in \mathbb{Z}$, where $t_{0}$ is an initial point, then $\left\{\varphi\left(t_{k}\right)\right\}$ is an S-almost automorphic sequence in $\mathbb{X}$.

Proof. Let $t_{k}^{j}=\delta_{-}\left(t_{k}, t_{k+j}\right), k, j \in \mathbb{Z}$. Obviously, from the definition of $\Pi^{ \pm}$, it is easy to know that $t_{k}^{j} \in \Pi^{ \pm}$. Since $\varphi \in P C_{r d}(\mathbb{T}, \mathbb{X})$ is an $S$-almost automorphic function and $\left\{t_{k}\right\} \subset \mathbb{T}$ is $S$-equipotentially almost automorphic, from Definitions 3 and 6 , for any sequence $\left\{s_{n}\right\} \subset \mathbb{Z}$, there exists a subsequence $\left\{s_{n}^{\prime}\right\}$ such that

$$
\begin{aligned}
\lim _{n \rightarrow \infty}\left(\varphi\left(t_{k+s_{n}^{\prime}}\right), \delta_{-}\left(t_{k}^{s_{n}^{\prime}}, T\right)\right) & =\lim _{n \rightarrow \infty}\left(\varphi\left(\delta_{+}\left(t_{k}^{s_{n}^{\prime}}, t_{k}\right)\right), \delta_{-}\left(t_{k}^{s_{n}^{\prime}}, T\right)\right) \\
& =\left(\varphi^{*}\left(t_{k}\right), T^{*}\right)=\left(\varphi\left(\delta_{+}\left(\gamma_{k}, t_{k}\right)\right), \delta_{-}\left(\gamma_{k}, T\right)\right) .
\end{aligned}
$$

and from $t_{k}^{s_{n}^{\prime}} \rightarrow \gamma_{k}(n \rightarrow \infty)$ for each $k$, we obtain

$$
\begin{aligned}
\lim _{n \rightarrow \infty}\left(\varphi^{*}\left(t_{k-s_{n}^{\prime}}\right), \delta_{+}\left(t_{k}^{s_{n}^{\prime}}, T^{*}\right)\right) & =\lim _{n \rightarrow \infty}\left(\varphi\left(\delta_{+}\left(\gamma_{k-s_{n}^{\prime}}, t_{k-s_{n}^{\prime}}\right)\right), \delta_{+}\left(t_{k}^{s_{n}^{\prime}}, \delta_{-}\left(\gamma_{k}, T\right)\right)\right) \\
& =\lim _{n \rightarrow \infty}\left(\varphi\left(\delta_{+}\left(\gamma_{k-s_{n}^{\prime}}, t_{k-s_{n}^{\prime}}\right)\right), \delta_{-}\left(\delta_{-}\left(t_{k}^{s_{n}^{\prime}}, \gamma_{k}\right), T\right)\right) \\
& =\left(\varphi\left(t_{k}\right), T\right) .
\end{aligned}
$$

Hence, $\left\{\varphi\left(t_{k}\right)\right\}$ is an $S$-almost automorphic sequence in $\mathbb{X}$. This completes the proof.

Denote $\Pi^{-}=\left\{s \in \Pi^{ \pm}: s \leq t_{0}\right\}$ and $\Pi^{+}=\left\{s \in \Pi^{ \pm}: s \geq t_{0}\right\}$.

To prove the following basic Lemma, we introduce notations $\delta_{-}(\tau, t):=\delta_{\tau_{-}}(t), \delta_{\tau_{-}}^{-k}(t)=$ $\delta_{-}\left(\tau, \delta_{\tau_{-}}^{-k-1}(t)\right), \delta_{+}(\tau, t):=\delta_{\tau}(t), \delta_{\tau}^{k}(t)=\delta_{+}\left(\tau, \delta_{\tau}^{k-1}(t)\right), k \in \mathbb{Z}^{+}$and $\delta_{\tau}^{0}\left(t_{0}\right)=\delta_{\tau-}^{0}\left(t_{0}\right)=t_{0}$.

Lemma 11. Let $\mathbb{T}$ be bi-direction S-CCTS under shifts $\delta_{ \pm}$and $\delta_{+}$be $\Delta$-differentiable to its second argument with $\delta_{+}^{\Delta}(s, \cdot)<\tilde{\delta}_{+}^{\Delta}\left(s \in \Pi^{-}\right)$, where $\tilde{\delta}_{+}^{\Delta}$ is a positive number. A necessary and sufficient condition for a bounded sequence $\left\{a_{n}\right\}$ to be in WPA $A_{0}^{S}(\mathbb{Z}, \rho)$ is that there exists a uniformly continuous function $f \in W P A A_{0}^{S}(\mathbb{T}, \rho)$ such that $f\left[\delta_{r(n)}^{n}\left(t_{0}\right)\right]=a_{n}, t_{0} \in \mathbb{T}, n \in \mathbb{Z}, \rho \in U_{B}$, where

$$
r(n)=\left\{\begin{array}{l}
r-\text { if } n<0 \\
t_{0} \quad \text { if } n=0 \\
r \quad \text { if } n>0
\end{array}\right.
$$

Proof. Necessity. We define a function

$$
f(t)=a_{n}+\left(t-\delta_{r(n)}^{n}\left(t_{0}\right)\right)\left(a_{n+1}-a_{n}\right), \delta_{r(n)}^{n}\left(t_{0}\right) \leq t<\delta_{r(n+1)}^{n+1}\left(t_{0}\right), t \in \mathbb{T}, n \in \mathbb{Z}, t_{0} \in \mathbb{T}^{*} .
$$

Since $f(t)$ has the bounded $\Delta$-derivative, it is uniformly continuous on $\mathbb{T}$. For each $j \in \mathbb{Z}$, note that

$$
\left|\delta_{r(j+1)}^{j+1}\left(t_{0}\right)-\delta_{r(j)}^{j}\left(t_{0}\right)\right|=\left|\delta_{\operatorname{sgn}(j)}\left(r, \delta_{r(j)}^{j}\left(t_{0}\right)\right)-\delta_{\operatorname{sgn}(j)}\left(t_{0}, \delta_{r(j)}^{j}\left(t_{0}\right)\right)\right| \leq\left|\tilde{\delta}_{+}^{\Delta}\right| \cdot r_{t_{0}},
$$

where $r_{t_{0}}:=\max \left\{\left|r-t_{0}\right|,\left|\delta_{-}\left(r, t_{0}\right)-t_{0}\right|\right\}$. 
We have $f \in W P A A_{0}^{S}(\mathbb{T}, \rho)$ since

$$
\begin{aligned}
& \frac{1}{m_{S}\left(\delta_{r}^{k-1}(r), \rho, t_{0}\right)} \int_{\delta_{r_{-}}^{-k}\left(t_{0}\right)}^{\delta_{r}^{k}\left(t_{0}\right)}\|f(s)\| \rho(s) \Delta s \\
& =\frac{1}{m_{S}\left(\delta_{r}^{k-1}(r), \rho, t_{0}\right)} \sum_{j=-k}^{k-1} \int_{\delta_{r(j)}^{j}\left(t_{0}\right)}^{\delta_{r(j+1)}^{j+1}\left(t_{0}\right)}\left\|a_{j}+\left(s-\delta_{r(j)}^{j}\left(t_{0}\right)\right)\left(a_{j+1}-a_{j}\right)\right\| \rho(s) \Delta s \\
& \leq \frac{1}{m_{S}\left(\delta_{r}^{k-1}(r), \rho, t_{0}\right) \underline{\rho}} \sum_{j=-k}^{k-1}\left(\left\|a_{j}\right\| \mu\left(t_{j}\right) \rho\left(t_{j}\right)\left|\tilde{\delta}_{-}^{\Delta}\right| \cdot r_{t_{0}}\right. \\
& \left.+\left\|a_{j+1}-a_{j}\right\| \int_{\delta_{r(j)}^{j}\left(t_{0}\right)}^{\delta_{r(j+1)}^{j+1}\left(t_{0}\right)}\left(s-\delta_{r(j)}^{j}\left(t_{0}\right)\right) \rho(s) \Delta s\right) \\
& \leq \frac{1}{\underline{\rho} m_{S}\left(\delta_{r}^{k-1}(r), \rho, t_{0}\right)} \sum_{j=-k}^{k-1}\left|\tilde{\delta}_{-}^{\Delta}\right| \cdot r_{t_{0}}\left\|a_{j}\right\| \mu\left(t_{j}\right) \rho\left(t_{j}\right)+\frac{\left(\left\|a_{k}\right\|+\left\|a_{-k}\right\|\right)\left(\left|\tilde{\delta}_{-}^{\Delta}\right| \cdot r_{t_{0}}\right)^{2}}{m_{S}\left(\delta_{r}^{k-1}(r), \rho, t_{0}\right)} \bar{\rho} \\
& \leq \frac{1}{\underline{\rho}_{t_{j} \in\left[\delta_{r_{-}}^{k}\left(t_{0}\right), \sigma_{r}^{k}\left(t_{0}\right)\right]_{\mathbb{T}^{*}}} \mu\left(t_{j}\right) \rho\left(t_{j}\right)} \sum_{j=-k}^{k-1}\left|\tilde{\delta}_{-}^{\Delta}\right| \cdot r_{t_{0}}\left\|f\left(t_{j}\right)\right\| \mu\left(t_{j}\right) \rho\left(t_{j}\right) \\
& +\frac{\left\|a_{k}\right\|+\left\|a_{-k}\right\|}{m_{S}\left(\delta_{r}^{k-1}(r), \rho, t_{0}\right)}\left(\left|\tilde{\delta}_{-}^{\Delta}\right| \cdot r_{t_{0}}\right)^{2} \bar{\rho} \\
& =\frac{1}{\underline{\rho} \sum_{j=-k}^{k-1} \mu\left(t_{j}\right) \rho\left(t_{j}\right)} \sum_{j=-k}^{k-1}\left|\tilde{\delta}_{-}^{\Delta}\right| \cdot r_{t_{0}}\left\|f\left(t_{j}\right)\right\| \mu\left(t_{j}\right) \rho\left(t_{j}\right) \\
& +\frac{\left\|a_{k}\right\|+\left\|a_{-k}\right\|}{m_{S}\left(\delta_{r}^{k-1}(r), \rho, t_{0}\right)}\left(\left|\tilde{\delta}_{-}^{\Delta}\right| \cdot r_{t_{0}}\right)^{2} \bar{\rho} \rightarrow 0, \text { as } k \rightarrow \infty,
\end{aligned}
$$

where $\rho=\inf _{t \in \mathbb{T}} \rho(t), \bar{\rho}=\sup _{t \in \mathbb{T}} \rho(t)$.

Sufficiency. Let $0<\varepsilon<1$, there exists $\delta^{*}>0$, for $t \in\left(t_{n}, t_{n}+\delta^{*}\right)_{\mathbb{T}}, n \in \mathbb{Z}$, such that

$$
\|f(t)\| \rho(t) \geq(1-\varepsilon)\left\|f\left(t_{n}\right)\right\| \rho\left(t_{n}\right), n \in \mathbb{Z} .
$$

Without loss of generality, let $t_{n} \geq t_{0}, t_{-n}<t_{0}, n \in \mathbb{Z}^{+}$and

$$
t_{-n}<t_{-n+1}<\ldots<t_{0}<t_{1}<\ldots<t_{n-1}<t_{n}
$$

there exists $r_{n}, r_{-n} \in\left(t_{0},+\infty\right)_{\Pi^{ \pm}}$such that $\delta_{+}\left(r_{n}, t_{0}\right)=t_{n}, \delta_{-}\left(r_{-n}, t_{0}\right)=t_{-n}$. Let $r_{n}^{\prime}=\max \left\{r_{n}, r_{-n}\right\} \in$ $\Pi^{ \pm}$. Therefore,

$$
\begin{aligned}
\int_{\delta_{-}\left(r_{n}^{\prime}, t_{0}\right)}^{\delta_{+}\left(r_{n}^{\prime}, t_{0}\right)}\|f(t)\| \rho(t) \Delta t & \geq \int_{\delta_{-}\left(r_{n}, t_{0}\right)}^{\delta_{+}\left(r_{n}, t_{0}\right)}\|f(t)\| \rho(t) \Delta t=\int_{t_{-n}}^{t_{n}}\|f(t)\| \rho(t) \Delta t \\
& \geq \sum_{j=-n}^{n-1} \int_{j_{j}}^{t_{j+1}}\|f(t)\| \rho(t) \Delta t \geq \sum_{j=-n}^{n-1} \int_{t_{j}+\delta^{*}}^{\sigma\left(t_{j}\right)}\|f(t)\| \rho(t) \Delta t \\
& \geq \sum_{j=-n}^{n-1}\left(\mu\left(t_{j}\right)-\delta^{*}\right)(1-\varepsilon)\left\|f\left(t_{j}\right)\right\| \rho\left(t_{j}\right) \\
& \geq(1-\varepsilon) \sum_{j=-n}^{n-1}\left(\mu\left(t_{j}\right)-\delta^{*}\right)\left\|f\left(t_{j}\right)\right\| \rho\left(t_{j}\right)
\end{aligned}
$$

so one can obtain

$$
\frac{1}{m_{S}\left(r_{n}^{\prime}, \rho, t_{0}\right)} \int_{\delta_{-}\left(r_{n}^{\prime}, t_{0}\right)}^{\delta_{+}\left(r_{n}^{\prime}, t_{0}\right)}\|f(t)\| \rho(t) \Delta t \geq(1-\varepsilon) \frac{1}{m_{S}\left(r_{n}^{\prime}, \rho, t_{0}\right)} \sum_{j=-n}^{n-1}\left(\mu\left(t_{j}\right)-\delta^{*}\right)\left\|f\left(t_{j}\right)\right\| \rho\left(t_{j}\right),
$$

it is easy to see that $r_{n}^{\prime}$ is increasing with respect to $n \in \mathbb{Z}^{+}$, one can find some $n_{0}>n$ such that

$$
m_{S}\left(r_{n}^{\prime}, \rho, t_{0}\right)=\int_{\delta_{-}\left(r_{n}^{\prime}, t_{0}\right)}^{\delta_{+}\left(r_{n}^{\prime}, t_{0}\right)} \rho(s) \Delta s \leq \sum_{t_{j} \in\left[\delta_{-}\left(r_{n_{0}}^{\prime}, t_{0}\right), \delta_{+}\left(r_{n_{0}}^{\prime}, t_{0}\right)\right]_{\mathbb{T}^{*}}} \mu\left(t_{j}\right) \rho\left(t_{j}\right)=\sum_{j=-n_{0}}^{n_{0}-1} \mu\left(t_{j}\right) \rho\left(t_{j}\right),
$$


from (9) and (10), we have

$$
\frac{1}{m_{S}\left(r_{n}^{\prime}, \rho, t_{0}\right)} \int_{\delta_{-}\left(r_{n}^{\prime}, t_{0}\right)}^{\delta_{+}\left(r_{n}^{\prime}, t_{0}\right)}\|f(t)\| \rho(t) \Delta t \geq(1-\varepsilon) \frac{1}{\sum_{j=-n_{0}}^{n_{0}-1} \mu\left(t_{j}\right) \rho\left(t_{j}\right)} \sum_{j=-n_{0}}^{n_{0}-1}\left(\mu\left(t_{j}\right)-\delta^{*}\right)\left\|f\left(t_{j}\right)\right\| \rho\left(t_{j}\right),
$$

noting that $n \rightarrow \infty$ implies $n_{0} \rightarrow \infty$, since $f \in W P A A_{0}^{S}(\mathbb{T}, \rho)$, it follows from the inequality (11) that $f\left(t_{n}\right)=a_{n} \in W P A A_{0}^{S}(\mathbb{Z}, \rho)$. This completes the proof.

By Lemma 11, we can straightly get the following theorem:

Theorem 5. Let $\mathbb{T}$ be bi-direction S-CCTS under shifts $\delta_{ \pm}$and $\delta_{+}$be $\Delta$-differentiable to its second argument with $\delta_{+}^{\Delta}(s, \cdot)<\tilde{\delta}_{+}^{\Delta}\left(s \in \Pi^{-}\right)$, where $\tilde{\delta}_{+}^{\Delta}$ is a positive number. A necessary and sufficient condition for a bounded sequence $\left\{a_{n}\right\}$ to be in $W P A A_{S}(\mathbb{Z}, \rho)$ is that there exists a uniformly continuous function $f \in W P A A_{S}(\mathbb{T}, \rho)$ such that $f\left[\delta_{r(n)}^{n}\left(t_{0}\right)\right]=a_{n}, t_{0} \in \mathbb{T}, n \in \mathbb{Z}, \rho \in U_{B}$.

Theorem 6. Let $\mathbb{T}$ be bi-direction S-CCTS under shifts $\delta_{ \pm}$and $\delta_{+}$be $\Delta$-differentiable to its second argument with $\delta_{+}^{\Delta}(s, \cdot)<\tilde{\delta}_{+}^{\Delta}$, where $\tilde{\delta}_{+}^{\Delta}$ is a positive number. Assume that $\rho \in U_{B}$ and the sequence of vector-valued functions $\left\{I_{k}\right\}_{k \in \mathbb{Z}}$ is weighted pseudo S-almost automorphic, i.e., for any $x \in \Omega,\left\{I_{k}(x), k \in \mathbb{Z}\right\}$ is weighted pseudo S-almost automorphic sequence. Suppose $\left\{I_{k}(x): k \in \mathbb{Z}, x \in K\right\}$ is bounded for every bounded subset $K \subseteq \Omega, I_{k}(x)$ is uniformly continuous in $x \in \Omega$ uniformly in $k \in \mathbb{Z}$. If $h \in W P A A_{S}(\mathbb{T}, \rho) \cap U P C(\mathbb{T}, \mathbb{X})$ such that $h(\mathbb{T}) \subset \Omega$, then $I_{k}\left(h\left(t_{k}\right)\right)$ is a weighted pseudo S-almost automorphic sequence.

Proof. Fix $h \in W P A A_{S}(\mathbb{T}, \rho) \cap U P C(\mathbb{T}, \mathbb{X})$, first we show $h\left(t_{k}\right)$ is weighted pseudo $S$-almost automorphic. Since $h=\phi_{1}+\phi_{2}$, where $\phi_{1} \in A A_{S}(\mathbb{T}, \mathbb{X}), \phi_{2} \in W P A A_{0}^{S}(\mathbb{T}, \rho)$. It follows from Lemma 10 that the sequence $\phi_{1}\left(t_{k}\right)$ is $S$-almost automorphic. To show $h\left(t_{k}\right)$ is weighted pseudo $S$-almost automorphic, we need to show that $\phi_{2}\left(t_{k}\right) \in W P A A_{0}^{S}(\mathbb{Z}, \rho)$. By the assumption, $h, \phi_{1} \in U P C(\mathbb{T}, \mathbb{X})$, so is $\phi_{2}$. Let $0<\varepsilon<1$, there exists $\delta^{*}>0$ such that for $t \in\left(t_{k}, t_{k}+\delta^{*}\right)_{\mathbb{T}}, k \in \mathbb{Z}$, we have

$$
\left\|\phi_{2}(t)\right\| \rho(t) \geq(1-\varepsilon)\left\|\phi_{2}\left(t_{k}\right)\right\| \rho\left(t_{k}\right), k \in \mathbb{Z} .
$$

Without loss of generality, let $t_{n} \geq t_{0}, t_{-n}<t_{0}, n \in \mathbb{Z}^{+}$, there exists $r_{n}, r_{-n} \in\left(t_{0}, \infty\right)_{\Pi^{ \pm}}$such that $\delta_{+}\left(r_{n}, t_{0}\right)=t_{n}, \delta_{-}\left(r_{-n}, t_{0}\right)=t_{-n}$. Let $r_{n}^{\prime}=\max \left\{r_{n}, r_{-n}\right\} \in \Pi^{ \pm}$. Therefore, repeating the proof of Lemma 11, we can obtain $h\left(t_{k}\right)$ is weighted pseudo $S$-almost automorphic.

Now, we show $I_{k}\left(h\left(t_{k}\right)\right)$ is weighted pseudo $S$-almost automorphic. Let

$$
\begin{aligned}
I(t, x)= & I_{k}(x)+\left(t-\delta_{r(k)}^{k}\left(t_{0}\right)\right)\left[I_{k+1}(x)-I_{k}(x)\right], \\
& \delta_{r(k)}^{k}\left(t_{0}\right) \leq t<\delta_{r(k+1)}^{k+1}\left(t_{0}\right), k \in \mathbb{Z}, r \in \Pi^{ \pm}, \\
\Phi_{0}(t) \quad= & h\left(t_{k}\right)+\left(t-\delta_{r(k)}^{k}\left(t_{0}\right)\right)\left[h\left(t_{k+1}\right)-h\left(t_{k}\right)\right], \\
& \delta_{r(k)}^{k}\left(t_{0}\right) \leq t<\delta_{r(k+1)}^{k+1}\left(t_{0}\right), k \in \mathbb{Z}, r \in \Pi^{ \pm} .
\end{aligned}
$$

Since $I_{k}, h\left(t_{k}\right)$ are two weighted pseudo $S$-almost automorphic, by Lemma 11 and Theorem 5 , we know that $I \in W P A A_{S}(\mathbb{T} \times \Omega, \rho), \Phi_{0} \in W P A A_{S}(\mathbb{T}, \rho)$. For every $t \in \mathbb{T}$, there exists a number $k \in \mathbb{Z}$ such that $\left|t-\delta_{r(k)}^{k}\left(t_{0}\right)\right| \leq r$,

$$
\begin{aligned}
\|I(t, x)\| & \leq\left\|I_{k}(x)\right\|+\left|t-\delta_{r(k)}^{k}\left(t_{0}\right)\right|\left[\left\|I_{k+1}(x)\right\|+\left\|I_{k}(x)\right\|\right] \\
& \leq(1+r)\left\|I_{k}(x)\right\|+r\left\|I_{k+1}(x)\right\| .
\end{aligned}
$$


Since $\left\{I_{k}(x): k \in \mathbb{Z}, x \in K\right\}$ is bounded for every bounded set $K \subseteq \Omega,\{I(t, x): t \in \mathbb{T}, x \in K\}$ is bounded for every bounded set $K \subseteq \Omega$. For every $x_{1}, x_{2} \in \Omega$, we have

$$
\begin{aligned}
\left\|I\left(t, x_{1}\right)-I\left(t, x_{2}\right)\right\| \leq & \left\|I_{k}\left(x_{1}\right)-I_{k}\left(x_{2}\right)\right\|+\left|t-\delta_{r(k)}^{k}\left(t_{0}\right)\right|\left[\left\|I_{k+1}\left(x_{1}\right)-I_{k+1}\left(x_{2}\right)\right\|\right. \\
& \left.+\left\|I_{k}\left(x_{1}\right)-I_{k}\left(x_{2}\right)\right\|\right] \\
\leq & (1+r)\left\|I_{k}\left(x_{1}\right)-I_{k}\left(x_{2}\right)\right\|+r\left\|I_{k+1}\left(x_{1}\right)-I_{k+1}\left(x_{2}\right)\right\| .
\end{aligned}
$$

Noting that $I_{k}(x)$ is uniformly continuous in $x \in \Omega$ uniformly in $k \in \mathbb{Z}$, we then get that $I(t, x)$ is uniformly continuous in $x \in \Omega$ for $t \in \mathbb{T}$. Then by Theorem $4, I\left(\cdot, \Phi_{0}(\cdot)\right) \in W P A A_{S}(\mathbb{T}, \mathbb{X})$. Again, using Lemma 11 and Theorem 5 , we have that $I\left(\delta_{r(k)}^{k}\left(t_{0}\right), \Phi_{0}\left(\delta_{r(k)}^{k}\left(t_{0}\right)\right)\right)$ is a weighted pseudo $S$-almost autmorphic sequence, that is, $I_{k}\left(h\left(t_{k}\right)\right)$ is weighted pseudo $S$-almost automorphic. This completes the proof.

From Theorem 6, one can easily get the following corollary:

Corollary 2. Let $\mathbb{T}$ be bi-direction S-CCTS under shifts $\delta_{ \pm}$and $\delta_{+}$be $\Delta$-differentiable to its second argument with $\delta_{+}^{\Delta}(s, \cdot)<\tilde{\delta}_{+}^{\Delta}$, where $\tilde{\delta}_{+}^{\Delta}$ is a positive number. Assume the sequence of vector-valued functions $\left\{I_{k}\right\}_{k \in \mathbb{Z}}$ is weighted pseudo S-almost automorphic, $\rho \in U_{B}$, if there is a number $L>0$ such that

$$
\left\|I_{k}(x)-I_{k}(y)\right\| \leq L\|x-y\|
$$

for all $x, y \in \Omega, k \in \mathbb{Z}$ and $h \in W P A A_{S}(\mathbb{T}, \rho) \cap U P C(\mathbb{T}, \rho)$ such that $h(\mathbb{T}) \subset \Omega$, then $I_{k}\left(h\left(t_{k}\right)\right)$ is a weighted pseudo $S$-almost automorphic sequence.

\section{Weighted Piecewise Pseudo S-Almost Automorphic Mild Solutions to the Impulsive $\Delta$-Evolution Equations}

In this section, we investigate the existence and exponential stability of a piecewise weighted pseudo $S$-almost automorphic mild solution to Equation (1). For this, we will provide a Lemma that will be used in our main results.

Lemma 12. Let $\mathbb{T}$ be bi-direction S-CCTS under shifts $\delta_{ \pm}$and $\delta_{+}$be $\Delta$-differentiable to its second argument with $\delta_{+}^{\Delta}(s, \cdot)<\tilde{\delta}_{+}^{\Delta}\left(s \in \Pi^{-}\right)$, where $\tilde{\delta}_{+}^{\Delta}$ is a positive number. Assume that $\omega \in \mathcal{R}^{+}$, for all $t \in \mathbb{T}, \alpha \in \Pi^{-}$, there exist constants $\beta_{1, \alpha}, \beta_{2, \alpha}>0$ such that

$$
\beta_{1, \alpha} \mu(t) \leq \mu\left(\delta_{+}(\alpha, t)\right) \leq \beta_{2, \alpha} \mu(t) .
$$

Then there exists positive constants $K^{*}$ and $\omega^{*}$ such that

$$
e_{\ominus \omega}\left(\delta_{+}(\alpha, t), \delta_{+}(\alpha, s)\right) \leq K^{*} e_{\ominus \omega^{*}}(t, s), t \geq s .
$$

Proof. Obviously, if $\mu=0, \mathbb{T}=\mathbb{R}$, the result holds. Assume that $\mu \not \equiv 0$. Since $\ominus \omega \in \mathcal{R}^{+}$, one has

$$
\begin{aligned}
& e_{\ominus \omega}\left(\delta_{+}(\alpha, t), \delta_{+}(\alpha, s)\right)=\exp \left\{-\int_{\delta_{+}(\alpha, s)}^{\delta_{+}(\alpha, t)} \frac{1}{\mu(\tau)} \ln \frac{1}{1-\mu(\tau) \omega} \Delta \tau\right\} \\
= & \exp \left\{-\int_{s}^{t} \frac{1}{\mu\left(\delta_{+}(\alpha, \tau)\right)} \ln \frac{1}{1-\mu\left(\delta_{+}(\alpha, \tau)\right) \omega} \delta_{+}^{\Delta}(\alpha, \tau) \Delta \tau\right\} .
\end{aligned}
$$


Hence, by the Inequality (12), we can obtain

$$
\begin{aligned}
& e_{\ominus \omega}\left(\delta_{+}(\alpha, t), \delta_{+}(\alpha, s)\right) \leq \exp \left\{-\int_{s}^{t} \frac{\tilde{\delta}_{+}^{\Delta}}{\beta_{2, \alpha} \mu(\tau)} \ln \frac{1}{1-\beta_{1, \alpha} \mu(\tau) \omega} \Delta \tau\right\} \\
& =\left\{\exp \left\{-\int_{s}^{t} \frac{\ln \left(1-\mu(\tau)\left(\ominus \beta_{1, \alpha} \omega\right)\right)}{\mu(\tau)}\right\}\right\}^{\frac{\frac{\delta_{+}}{\beta_{2, \alpha}}}{2}} \text {. }
\end{aligned}
$$

Therefore, there exists a positive constant $K^{*}>0$ such that

$$
e_{\ominus \omega}\left(\delta_{+}(\alpha, t), \delta_{+}(\alpha, s)\right)=\left[e_{\ominus \beta_{1, \alpha} \omega}(t, s)\right]^{\frac{\tilde{\delta}_{+}^{4}}{\beta_{2, \alpha}}} \leq K^{*} e_{\ominus \omega^{*}}(t, s),
$$

where $\omega^{*}=\beta_{1, \alpha} \omega$. This completes the proof.

Remark 8. It is easy to observe that if $\mu(t)$ is bounded, then there exists a sufficiently small constant $\beta_{1, \alpha}>0$ and a sufficiently large constant $\beta_{2, \alpha}>0$ such that (12) is valid. Therefore, Lemma 12 holds when $\mathbb{T}$ is an almost periodic time scale from [33].

For the time scale from (1) in Example 1, we can obtain that

$$
\begin{aligned}
& \mu\left(\delta_{+}(\alpha, t)\right)=\left\{\begin{array}{l}
q^{2} \alpha t-\alpha t, t>0, \\
\frac{t}{\alpha q^{2}}-\frac{t}{\alpha}, t<0,
\end{array} \quad \mu(t)=\left\{\begin{array}{l}
q^{2} t-t, t>0, \\
\frac{t}{q^{2}}-t, t<0,
\end{array}\right.\right. \\
& \left\{\begin{array}{l}
\beta_{1, \alpha}=\beta_{2, \alpha}=\alpha, t>0, \\
\beta_{1, \alpha}=\beta_{2, \alpha}=\frac{1}{\alpha}, t<0 .
\end{array}\right.
\end{aligned}
$$

Hence, for all $t \in \mathbb{T}^{*}, \alpha<1$, we can obtain $\alpha \mu(t) \leq \mu\left(\delta_{+}(\alpha, t)\right) \leq \frac{1}{\alpha} \mu(t)$.

Consider the impulsive linear $\Delta$-evolution equation

$$
x^{\Delta}=A(t) x^{\sigma}, t \in \mathbb{T},
$$

where $A: \mathbb{T} \rightarrow B(\mathbb{X})$ is a linear operator in the Banach space $\mathbb{X}$. We denote by $B(\mathbb{X}, \mathbb{Y})$ the Banach space of all bounded linear operators from $\mathbb{X}$ to $\mathbb{Y}$. This is simply denoted as $B(\mathbb{X})$ when $\mathbb{X}=\mathbb{Y}$.

Definition 8 ([11]). $T(t, s): \mathbb{T} \times \mathbb{T} \rightarrow B(\mathbb{X})$ is called the linear evolution operator associated to (13) if $T(t, s)$ satisfies the following conditions:

(1) $T(s, s)=\mathrm{Id}$, where Id denotes the identity operator in $\mathbb{X}$;

(2) $T(t, s) T(s, r)=T(t, r)$;

(3) the mapping $(t, s) \rightarrow T(t, s) x$ is continuous for any fixed $x \in \mathbb{X}$.

Definition 9. A function $x: \mathbb{T} \rightarrow \mathbb{X}$ is called a mild solution of Equation (1) if for any $t \in \mathbb{T}, t>c, c \neq$ $t_{k}, k \in \mathbb{Z}$, one has

$$
x(t)=T(t, c) x(c)+\int_{c}^{t} T(t, s) f(s, x(s)) \Delta s+\sum_{c<t_{k}<t} T\left(t, t_{k}\right) I_{k}\left(x\left(t_{k}\right)\right) .
$$

In the following, consider (1) with the following assumptions:

$\left(H_{1}\right)$ Let $\mathbb{T}$ be bi-direction S-CCTS under shifts $\delta_{ \pm}$and $\delta_{+}$be $\Delta$-differentiable to its second argument with $\delta_{+}^{\Delta}(s, \cdot)<\tilde{\delta}_{+}^{\Delta}\left(s \in \Pi^{-}\right)$, where $\tilde{\delta}_{+}^{\Delta}$ is a positive number. 
$\left(H_{2}\right)$ The family $\{A(t): t \in \mathbb{T}\}$ of operators in $\mathbb{X}$ generates an $S$-exponentially stable evolution system $\{T(t, s): t \geq s\}$, i.e., there exist $K_{0}>1$ and $\omega>0$ such that

$$
\|T(t, s)\|_{B(\mathbb{X})} \leq \frac{K_{0}}{1+\mu(t) \omega} e_{\ominus \omega}(t, s), t \geq s, \omega \in \mathcal{R}^{+} .
$$

$\left(H_{3}\right) \quad f=g+\phi \in W P A A_{S}(\mathbb{T}, \rho)$, where $\rho \in U_{B}$ and $f(t, \cdot)$ is uniformly continuous in each bounded subset of $\Omega$ uniformly in $t \in \mathbb{T}$; $I_{k}$ is a weighted pseudo $S$-almost periodic sequence, $I_{k}(x)$ is uniformly continuous in $x \in \Omega$ uniformly in $k \in \mathbb{Z}, \inf _{k \in \mathbb{Z}} t_{k}^{1}=\delta_{-}\left(t_{k}, t_{k+1}\right)=\theta>\max \left\{t_{0}, 0\right\}$, where $t_{0}$ is the initial point.

Remark 9. In the assumption $\left(H_{2}\right)$, let $\mathbb{T}=\mathbb{R}$, Equation (14) turns into

$$
\|T(t, s)\|_{B(\mathbb{X})} \leq K_{0} e^{-\omega(t-s)}, t \geq s .
$$

Let $\mathbb{T}=h \mathbb{Z}, h>0$ and $K^{p}=\frac{K_{0}}{1+\omega}$, it becomes

$$
\|T(t, s)\|_{B(\mathbb{X})} \leq K^{p}\left(1-\frac{\omega h}{1+\omega}\right)^{\frac{t-s}{h}}, t \geq s .
$$

Moreover, let the time scale $\mathbb{T}$ be the quantum time scale $q^{\mathbb{Z}}=\left\{q^{n}: q>1, n \in \mathbb{Z}\right\}$, then

$$
\|T(t, s)\|_{B(\mathbb{X})} \leq \frac{K_{0}}{1+\omega(q-1) t} \prod_{s^{*} \in[s, t)} \frac{1}{1+(q-1) \omega s^{*}} .
$$

Let $\mu=\inf _{t \in \mathbb{T}} \mu(t)$ and $\bar{\mu}=\sup _{t \in \mathbb{T}} \mu(t)$. To investigate the existence and uniqueness of a weighted piecewise pseudo $S$-almost automorphic mild solution to Equation (1), we need the following Lemma:

Lemma 13. Assume $v \in A A_{S}(\mathbb{T}, \mathbb{X}), \omega \in \mathcal{R}^{+}$and $\left(H_{1}\right)-\left(H_{3}\right)$ are satisfied. If $u: \mathbb{T} \rightarrow \mathbb{X}$ is defined by

$$
u_{0}(t)=\int_{-\infty}^{t} T(t, s) v(s) \Delta s+\sum_{t_{k}<t} T\left(t, t_{k}\right) I_{k}\left(v\left(t_{k}\right)\right), t \geq s,
$$

then $u_{0}(\cdot) \in A A_{S}(\mathbb{T}, \mathbb{X})$.

Proof. Let $\left\{s_{n}\right\}_{n=1}^{\infty} \subset \Pi^{-}$. Since $v$ is almost automorphic, there exists a subsequence $\left\{\tau_{n}\right\}_{n=1}^{\infty} \subset$ $\left\{s_{n}\right\}_{n=1}^{\infty}$ such that $h(t):=\lim _{n \rightarrow \infty} v\left(\delta_{+}\left(\tau_{n}, t\right)\right)$ is well defined for each $t \in \mathbb{T}$.

Now, we consider

$$
\begin{aligned}
u\left(\delta_{+}\left(\tau_{n}, t\right)\right) & =\int_{-\infty}^{\delta_{+}\left(\tau_{n}, t\right)} T\left(\delta_{+}\left(\tau_{n}, t\right), s\right) v(s) \Delta \\
s & =\int_{-\infty}^{t} T\left(\delta_{+}\left(\tau_{n}, t\right), \delta_{+}\left(\tau_{n}, s\right)\right) v\left(\delta_{+}\left(\tau_{n}, s\right)\right) \delta_{+}^{\Delta}\left(\tau_{n}, s\right) \Delta s \\
& =\int_{-\infty}^{t} T\left(\delta_{+}\left(\tau_{n}, t\right), \delta_{+}\left(\tau_{n}, s\right)\right) \delta_{+}^{\Delta}\left(\tau_{n}, s\right) v_{n}(s) \Delta s,
\end{aligned}
$$

where $v_{n}(s)=v\left(\delta_{+}\left(\tau_{n}, s\right)\right), n=1,2, \ldots$

Since $\omega \in \mathcal{R}^{+}$, one can choose sufficiently small constant $\beta_{1}>0$ such that $\omega^{*}=\beta_{1} \omega$ is also positive regressive. Further, noting that $e_{\ominus \omega^{*}}(t, s)\left(1+\mu(s) \omega^{*}\right)=e_{\ominus \omega^{*}}(t, \sigma(s))$, by $\left(H_{1}\right)$ and Lemma 12, we have 


$$
\begin{aligned}
\left\|u\left(\delta_{+}\left(\tau_{n}, t\right)\right)\right\| & \leq \int_{-\infty}^{t}\left\|T\left(\delta_{+}\left(\tau_{n}, t\right), \delta_{+}\left(\tau_{n}, s\right)\right) \delta_{+}^{\Delta}\left(\tau_{n}, s\right) v_{n}(s)\right\| \Delta s \\
& \leq \int_{-\infty}^{t} \tilde{\delta}_{+}^{\Delta} \frac{K_{0}}{1+\mu(t) \omega} e_{\ominus \omega}\left(\delta_{+}\left(\tau_{n}, t\right), \delta_{+}\left(\tau_{n}, s\right)\right)\left\|v_{n}(s)\right\| \Delta s \\
& \leq \frac{K_{0} K^{*} \tilde{\delta}_{+}^{\Delta}}{1+\underline{\mu} \omega} \int_{-\infty}^{t} e_{\ominus \omega^{*}}(t, s)\left\|v_{n}(s)\right\| \Delta s \\
& \leq \frac{K_{0} K^{*}\|v\|_{\infty} \tilde{\delta}_{+}^{\Delta}}{1+\underline{\mu} \omega} \int_{-\infty}^{t} \frac{1}{1+\mu \omega^{*}} e_{\ominus \omega^{*}}(t, \sigma(s)) \Delta s \\
& =\frac{K_{0} K^{*}\|v\|_{\infty} \tilde{\delta}_{+}^{\Delta}}{-\omega^{*}(1+\underline{\mu} \omega)}\left[e_{\ominus \omega^{*}}(t,-\infty)-e_{\ominus \omega^{*}}(t, t)\right]=\frac{K_{0} K^{*}\|v\|_{\infty} \tilde{\delta}_{+}^{\Delta}}{\omega^{*}(1+\underline{\mu} \omega)} .
\end{aligned}
$$

Therefore, by the condition $\left(H_{1}\right),\left(H_{2}\right)$, we have $T\left(\delta_{+}\left(\tau_{n}, t\right), \delta_{+}\left(\tau_{n}, s\right)\right) \rightarrow T^{*}(t, s), n \rightarrow \infty$. Furthermore, it is easy to see that $v_{n}(s) \rightarrow h(s)$ as $n \rightarrow \infty, \forall s \in \mathbb{T}$ and for any $t \geq s$, by Lebesgue's dominated convergence theorem, we get $\lim _{n \rightarrow \infty} u\left(\delta_{+}\left(\tau_{n}, t\right)\right)=\int_{-\infty}^{t} T^{*}(t, s) \delta_{*}(s) h(s) \Delta s$.

Moreover, we consider

$$
\begin{aligned}
u^{\prime}\left(\delta_{+}\left(\tau_{n}, t\right)\right) & :=\sum_{t_{k}<\delta_{+}\left(\tau_{n}, t\right)} T\left(\delta_{+}\left(\tau_{n}, t\right), t_{k}\right) I_{k}\left(v_{k}\left(t_{k}\right)\right) \\
& =\sum_{t_{k}<t} T\left(\delta_{+}\left(\tau_{n}, t\right), \delta_{+}\left(\tau_{n}, t_{k}\right)\right) I_{k}\left(v\left(\delta_{+}\left(\tau_{n}, t_{k}\right)\right)\right) \\
& =\sum_{t_{k}<t} T\left(\delta_{+}\left(\tau_{n}, t\right), \delta_{+}\left(\tau_{n}, t_{k}\right)\right) I_{k}\left(v_{k n}\right),
\end{aligned}
$$

where $v\left(\delta_{+}\left(\tau_{n}, t_{k}\right)\right):=v_{k n}$. By Lemma 12, we can get

$$
\begin{aligned}
\left\|u^{\prime}\left(\delta_{+}\left(\tau_{n}, t\right)\right)\right\| & =\left\|\sum_{t_{k}<\delta_{+}\left(\tau_{n}, t\right)} T\left(\delta_{+}\left(\tau_{n}, t\right), t_{k}\right) I_{k}\left(v_{k}\left(t_{k}\right)\right)\right\| \\
& =\left\|\sum_{t_{k}<t} T\left(\delta_{+}\left(\tau_{n}, t\right), \delta_{+}\left(\tau_{n}, t_{k}\right)\right) I_{k}\left(v_{k n}\right)\right\| \\
& \leq \frac{I K_{0}}{1+\underline{\mu} \omega} \sum_{t_{k}<t} e_{\ominus \omega}\left(\delta_{+}\left(\tau_{n}, t\right), \delta_{+}\left(\tau_{n}, t_{k}\right)\right) \\
& \leq \frac{I K_{0} K^{*}}{1+\underline{\mu} \omega} \sum_{t_{k}<t} e_{\ominus \omega^{*}}\left(t, t_{k}\right) \leq \frac{I K_{0} K^{*}}{1+\underline{\mu \omega}} \frac{1}{1-e_{\ominus \omega^{*}}\left(\theta, t_{0}\right)},
\end{aligned}
$$

where $\sup _{k \in \mathbb{Z}} e_{\ominus \omega^{*}}\left(t_{k+1}, t_{k}\right):=e_{\ominus \omega^{*}}\left(\theta, t_{0}\right)$.

Since $v \in A A_{S}(\mathbb{T}, \mathbb{X}), v_{k n} \rightarrow h\left(t_{k}\right), n \rightarrow \infty, \forall k \in \mathbb{Z}$. Hence, for any $t>t_{k}, k \in \mathbb{Z}$, by Lebesgue's dominated convergence theorem, we get $\lim _{n \rightarrow \infty} u^{\prime}\left(\delta_{+}\left(\tau_{n}, t\right)\right)=\sum_{t_{k}<t} T^{*}\left(t, t_{k}\right) I_{k}\left(h\left(t_{k}\right)\right)$. So we have $\lim _{n \rightarrow \infty} u_{0}\left(\delta_{+}\left(\tau_{n}, t\right)\right)=\lim _{n \rightarrow \infty} u\left(\delta_{+}\left(\tau_{n}, t\right)\right)+\lim _{n \rightarrow \infty} u^{\prime}\left(\delta_{+}\left(\tau_{n}, t\right)\right)$ is well defined for each $t \in \mathbb{T}^{*}$. Therefore, $u_{0}(\cdot) \in A A_{S}(\mathbb{T}, \mathbb{X})$. This completes the proof.

Theorem 7. Assume that $\left(H_{1}\right)-\left(H_{3}\right)$ are satisfied. Let $f(\cdot, \vartheta(\cdot)) \in W P A A_{S}(\mathbb{T}, \rho)$, where $\vartheta \in$ $W P A A_{S}(\mathbb{T}, \rho)$ and $\{T(t, s), t \geq s\}$ is exponentially stable, $\rho \in U_{B}$. Then

$$
F(\cdot):=\int_{-\infty}^{(\cdot)} T(\cdot, s) f(s, \vartheta(s)) \Delta s+\sum_{t_{k}<\cdot} T\left(\cdot, t_{k}\right) I_{k}\left(\vartheta\left(t_{k}\right)\right) \in W P A A_{S}(\mathbb{T}, \rho) .
$$


Proof. Fix $\vartheta \in W P A A_{S}(\mathbb{T}, \mathbb{X})$, then we have $f(\cdot, \vartheta(\cdot))=\phi_{1}(\cdot)+\phi_{2}(\cdot)$, where $\phi_{1} \in A A_{S}(\mathbb{T}, \mathbb{X})$, $\phi_{2} \in W P A A_{0}^{S}(\mathbb{T}, \mathbb{X})$, so

$$
\int_{-\infty}^{t} T(t, s) f(s, \vartheta(s)) \Delta s=\int_{-\infty}^{t} T(t, s) \phi_{1}(s) \Delta s+\int_{-\infty}^{t} T(t, s) \phi_{2}(s) \Delta s:=I_{1}(t)+I_{2}(t)
$$

and

$$
\sum_{t_{k}<t} T\left(t, t_{k}\right) I_{k}\left(\vartheta\left(t_{k}\right)\right)=\sum_{t_{k}<t} T\left(t, t_{k}\right) \beta_{k}+\sum_{t_{k}<t} T\left(t, t_{k}\right) \gamma_{k}:=\mathrm{Y}_{1}(t)+\mathrm{Y}_{2}(t)
$$

By Lemma 13, we can easily see that $I_{1}, \mathrm{Y}_{1} \in A A_{\mathcal{S}}(\mathbb{T}, \mathbb{X})$.

Moreover, we have

$$
\begin{aligned}
& \frac{1}{m_{S}\left(r, \rho, t_{0}\right)} \int_{\delta_{-}\left(r, t_{0}\right)}^{\delta_{+}\left(r, t_{0}\right)}\left\|I_{2}(t)\right\| \Delta t \\
= & \frac{1}{m_{S}\left(r, \rho, t_{0}\right)} \int_{\delta_{-}\left(r, t_{0}\right)}^{\delta_{+}\left(r, t_{0}\right)}\left\|\int_{-\infty}^{t} T(t, s) \phi_{2}(s) \Delta s\right\| \Delta t \\
\leq & \frac{1}{m_{S}\left(r, \rho, t_{0}\right)} \int_{\delta_{-}\left(r, t_{0}\right)}^{\delta_{+}\left(r, t_{0}\right)} \Delta t \int_{-\infty}^{t} K_{0} e_{\ominus \omega}(t, s)\left\|\phi_{2}(s)\right\| \Delta s \\
= & \frac{1}{m_{S}\left(r, \rho, t_{0}\right)} \int_{\delta_{-}\left(r, t_{0}\right)}^{\delta_{+}\left(r, t_{0}\right)} \Delta t\left(\int_{-\infty}^{\delta_{-}\left(r, t_{0}\right)} K_{0} e_{\ominus \omega}(t, s)\left\|\phi_{2}(s)\right\| \Delta s\right. \\
& \left.+\int_{\delta_{-}\left(r, t_{0}\right)}^{t} K_{0} e_{\ominus \omega}(t, s)\left\|\phi_{2}(s)\right\| \Delta s\right) \\
= & \frac{1}{m_{S}\left(r, \rho, t_{0}\right)} \int_{-\infty}^{\delta_{-}\left(r, t_{0}\right)}\left\|\phi_{2}(s)\right\| \Delta s \int_{\delta_{-}\left(r, t_{0}\right)}^{\delta_{+}\left(r, t_{0}\right)} K_{0} e_{\ominus \omega}(t, s) \Delta t \\
& +\frac{1}{m_{S}\left(r, \rho, t_{0}\right)} \int_{\delta_{-}\left(r, t_{0}\right)}^{\delta_{+}\left(r, t_{0}\right)}\left\|\phi_{2}(s)\right\| \Delta s \int_{s}^{\delta_{+}\left(r, t_{0}\right)} K_{0} e_{\ominus \omega}(t, s) \Delta t:=I_{1}^{0}+I_{2}^{0} .
\end{aligned}
$$

Then

$$
\begin{aligned}
I_{1}^{0}= & \frac{1}{m_{S}\left(r, \rho, t_{0}\right)} \int_{-\infty}^{\delta_{-}\left(r, t_{0}\right)}\left\|\phi_{2}(s)\right\| \Delta s \int_{\delta_{-}\left(r, t_{0}\right)}^{\delta_{+}\left(r, t_{0}\right)} \frac{K_{0}}{1+\mu(t) \omega} e_{\ominus \omega}(t, s) \Delta t \\
= & \frac{1}{m_{S}\left(r, \rho, t_{0}\right)} \int_{-\infty}^{\delta_{-}\left(r, t_{0}\right)}\left\|\phi_{2}(s)\right\| \Delta s \int_{\delta_{-}\left(r, t_{0}\right)}^{\delta_{+}\left(r, t_{0}\right)} K_{0} e_{\ominus \omega}(\sigma(t), s) \Delta t \\
\leq & \frac{K_{0}}{m_{S}\left(r, \rho, t_{0}\right)} \int_{-\infty}^{\delta_{-}\left(r, t_{0}\right)}\left\|\phi_{2}(s)\right\| \Delta s \int_{\delta_{-}\left(r, t_{0}\right)}^{\delta_{+}\left(r, t_{0}\right)} e_{\omega}(s, \sigma(t)) \Delta t \\
= & \frac{1}{m_{S}\left(r, \rho, t_{0}\right)} \frac{K_{0}}{\omega} \int_{-\infty}^{\delta_{-}\left(r, t_{0}\right)}\left\|\phi_{2}(s)\right\|\left[e_{\omega}\left(s, \delta_{-}\left(r, t_{0}\right)\right)-e_{\omega}\left(s, \delta_{+}\left(r, t_{0}\right)\right)\right] \Delta s \\
\leq & \frac{1}{m_{S}\left(r, \rho, t_{0}\right)} \frac{K_{0}}{\omega}\left\|\phi_{2}\right\|\left(\int_{-\infty}^{\delta_{-}\left(r, t_{0}\right)} e_{\ominus \omega}\left(\delta_{-}\left(r, t_{0}\right), s\right) \Delta s\right. \\
& \left.-\int_{-\infty}^{\delta_{-}\left(r, t_{0}\right)} e_{\ominus \omega}\left(\delta_{+}\left(r, t_{0}\right), s\right) \Delta s\right) \\
\leq & \frac{1}{m_{S}\left(r, \rho, t_{0}\right)} \frac{-K_{0}}{\omega}\left\|\phi_{2}\right\|\left(e_{\ominus \omega}\left(\delta_{-}\left(r, t_{0}\right),-\infty\right)-e_{\ominus \omega}\left(\delta_{-}\left(r, t_{0}\right), \delta_{-}\left(r, t_{0}\right)\right)\right. \\
& \left.-e_{\ominus \omega}\left(\delta_{+}\left(r, t_{0}\right),-\infty\right)+e_{\ominus \omega}\left(\delta_{+}\left(r, t_{0}\right), \delta_{-}\left(r, t_{0}\right)\right)\right) \rightarrow 0 \text { as } r \rightarrow \infty,
\end{aligned}
$$


and

$$
\begin{aligned}
I_{2}^{0} & =\frac{1}{m_{S}\left(r, \rho, t_{0}\right)} \int_{\delta_{-}\left(r, t_{0}\right)}^{\delta_{+}\left(r, t_{0}\right)}\left\|\phi_{2}(s)\right\| \Delta s \int_{s}^{\delta_{+}\left(r, t_{0}\right)} \frac{K_{0}}{1+\mu(t) \omega} e_{\ominus \omega}(t, s) \Delta t \\
& \leq \frac{1}{m_{S}\left(r, \rho, t_{0}\right)} \int_{\delta_{-}\left(r, t_{0}\right)}^{\delta_{+}\left(r, t_{0}\right)}\left\|\phi_{2}(s)\right\| \Delta s \int_{s}^{\delta_{+}\left(r, t_{0}\right)} K_{0} e_{\ominus \omega}(\sigma(t), s) \Delta t \\
& \leq \frac{K_{0}}{m_{S}\left(r, \rho, t_{0}\right)} \int_{\delta_{-}\left(r, t_{0}\right)}^{\delta_{+}\left(r, t_{0}\right)}\left\|\phi_{2}(s)\right\| \Delta s \int_{s}^{\delta_{+}\left(r, t_{0}\right)} e_{\omega}(s, \sigma(t)) \Delta t \\
& =\frac{1}{m_{S}\left(r, \rho, t_{0}\right)} \frac{K_{0}}{\omega} \int_{\delta_{-}\left(r, t_{0}\right)}^{\delta_{+}\left(r, t_{0}\right)}\left\|\phi_{2}(s)\right\|\left[e_{\omega}(s, s)-e_{\omega}\left(s, \delta_{+}\left(r, t_{0}\right)\right)\right] \Delta s \\
& \leq \frac{1}{m_{S}\left(r, \rho, t_{0}\right)} \frac{K_{0}}{\omega} \int_{\delta_{-}\left(r, t_{0}\right)}^{\delta_{+}\left(r, t_{0}\right)}\left\|\phi_{2}(s)\right\| \Delta s .
\end{aligned}
$$

Since $\phi_{2} \in W P A A_{0}^{S}(\mathbb{T}, \rho)$, we have $\lim _{r \rightarrow \infty} \frac{1}{m_{S}\left(r, \rho, t_{0}\right)} \int_{\delta_{-}\left(r, t_{0}\right)}^{\delta_{+}\left(r, t_{0}\right)}\left\|\phi_{2}(s)\right\| \Delta s=0$. Hence, $\lim _{r \rightarrow \infty} I_{2}^{0}=0$. It remains to show $\mathrm{Y}_{2} \in W P A A_{0}^{S}(\mathbb{T}, \rho)$. For any $r>0$, there exist $i(r), j(r)$ such that

$$
t_{i(r)-1}<\delta_{-}\left(r, t_{0}\right) \leq t_{i(r)}<\ldots<t_{j(r)} \leq \delta_{+}\left(r, t_{0}\right)<t_{j(r)+1} .
$$

Since $\gamma_{k} \in W P A A_{0}^{S}(\mathbb{Z}, \rho), M_{\gamma_{k}}=\sup _{k \in \mathbb{Z}}\left\|\gamma_{k}\right\|<\infty$, noting that for $a \in \mathbb{T}, e_{\ominus \omega}(t, a)=(1+$ $\mu(t) \omega) e_{\omega}(a, \sigma(t))$, then we can obtain

$$
\begin{aligned}
& \frac{1}{m_{S}\left(r, \rho, t_{0}\right)} \int_{\delta_{-}\left(r, t_{0}\right)}^{\delta_{+}\left(r, t_{0}\right)}\left\|\mathrm{Y}_{2}(t)\right\| \Delta t=\frac{1}{m_{S}\left(r, \rho, t_{0}\right)} \int_{\delta_{-}\left(r, t_{0}\right)}^{\delta_{+}\left(r, t_{0}\right)}\left\|\sum_{t_{k}<t} T\left(t, t_{k}\right) \gamma_{k}\right\| \Delta t \\
\leq & \frac{1}{m_{S}\left(r, \rho, t_{0}\right)} \int_{\delta_{-}\left(r, t_{0}\right)}^{\delta_{+}\left(r, t_{0}\right)} \sum_{t_{k}<t} \frac{K_{0}}{1+\mu(t) \omega} e_{\ominus \omega}\left(t, t_{k}\right)\left\|\gamma_{k}\right\| \Delta t \\
\leq & \frac{1}{m_{S}\left(r, \rho, t_{0}\right)} \sum_{t_{k}<\delta_{-}\left(r, t_{0}\right)} e_{\ominus \omega}\left(\delta_{-}\left(r, t_{0}\right), t_{k}\right)\left\|\gamma_{k}\right\| \\
& \times \int_{\delta_{-}\left(r, t_{0}\right)}^{\delta_{+}\left(r, t_{0}\right)} \frac{K_{0}}{1+\mu(t) \omega} e_{\ominus \omega}\left(t, \delta_{-}\left(r, t_{0}\right)\right) \Delta t \\
& +\frac{1}{m_{S}\left(r, \rho, t_{0}\right)} \sum_{\delta_{-}\left(r, t_{0}\right)<t_{k}<t<\delta_{+}\left(r, t_{0}\right)} e_{\ominus \omega}\left(t, t_{k}\right)\left\|\gamma_{k}\right\| \int_{\delta_{-}\left(r, t_{0}\right)}^{\delta_{+}\left(r, t_{0}\right)} \frac{K_{0}}{1+\mu(t) \omega} e_{\ominus \omega}\left(\sigma(t), t_{k}\right) \Delta t \\
\leq & \frac{1}{m_{\left(r, \rho, t_{0}\right)}} \sum_{t_{k}<\delta_{-}\left(r, t_{0}\right)} \frac{K_{0}}{\omega} M_{\gamma_{k}} e_{\ominus \omega}\left(\delta_{-}\left(r, t_{0}\right), t_{k}\right) \\
& +\frac{1}{m_{S}\left(r, \rho, t_{0}\right)} \sum_{\delta_{-}\left(r, t_{0}\right)<t_{k}<t<\delta_{+}\left(r, t_{0}\right)} \frac{K_{0}}{\omega}\left\|\gamma_{k}\right\| e_{\ominus \omega}\left(t, t_{k}\right) \\
\leq & \frac{1}{m_{S}\left(r, \rho, t_{0}\right)} \frac{K_{0} M_{\gamma_{k}}}{\omega} \frac{1}{1-e_{\ominus \omega}\left(\theta, t_{0}\right)}+\frac{K_{0}}{\omega} \frac{1}{m_{S}\left(r, \rho, t_{0}\right)} \sum_{k=i(r)}^{j(r)}\left\|\gamma_{k}\right\| \frac{1}{1-e_{\ominus \omega}\left(\theta, t_{0}\right)} .
\end{aligned}
$$

Since $\gamma_{k} \in W P A A_{0}^{S}(\mathbb{Z}, \rho)$, for $r \rightarrow \infty, m_{S}(r, \rho) \rightarrow \infty$, we have

$$
\lim _{r \rightarrow \infty} \frac{1}{m_{S}\left(r, \rho, t_{0}\right)} \sum_{k=i(r)}^{j(r)}\left\|\gamma_{k}\right\|=\lim _{r \rightarrow \infty} \frac{1}{\sum_{k=i(r)}^{j(r)} \rho\left(t_{k}\right) \mu\left(t_{k}\right)} \sum_{k=i(r)}^{j(r)}\left\|\gamma_{k}\right\|=0 .
$$

Clearly, as $r \rightarrow \infty$, one has

$$
\frac{1}{m_{S}\left(r, \rho, t_{0}\right)} \frac{K_{0} M_{\gamma_{k}}}{\omega} \frac{1}{1-e_{\ominus \omega}\left(\theta, t_{0}\right)} \rightarrow 0
$$


Hence

$$
\lim _{r \rightarrow \infty} \frac{1}{m_{S}\left(r, \rho, t_{0}\right)} \int_{\delta_{-}\left(r, t_{0}\right)}^{\delta_{+}\left(r, t_{0}\right)}\left\|\mathrm{Y}_{2}(t)\right\| \Delta t=0
$$
proof.

Thus, $\sum_{t_{k}<\cdot} T\left(\cdot, t_{k}\right) I_{k}\left(\vartheta\left(t_{k}\right)\right) \in W P A A_{0}^{S}(\mathbb{T}, \rho)$, then $F(\cdot) \in W P A A_{S}(\mathbb{T}, \rho)$. This completes the

Theorem 8. Assume $\left(H_{1}\right)-\left(H_{3}\right)$ are satisfied and the following conditions hold:

$\left(A_{1}\right)$ The family $\{A(t): t \in \mathbb{T}\}$ of operators in $\mathbb{X}$ generates an $\delta$-exponentially stable evolution system $\{T(t, s): t \geq s\}$, i.e., there exist $K_{0}>1$ and $\omega>0$ such that

$$
\|T(t, s)\|_{B(\mathbb{X})} \leq \frac{K_{0}}{1+\mu(t) \omega} e_{\ominus \omega}(t, s), t \geq s .
$$

$\left(A_{2}\right) f \in W P A A_{S}(\mathbb{T} \times \Omega, \rho)$, and $f$ satisfies the Lipschitz condition with respect to the second argument, i.e.,

$$
\|f(t, x)-f(t, y)\| \leq L_{1}\|x-y\|, t \in \mathbb{T}, x, y \in \Omega,
$$

$\left(A_{3}\right) \quad I_{k}$ is a weighted pseudo S-almost periodic sequence, and there exists a number $L_{2}>0$ such that

$$
\left\|I_{k}(x)-I_{k}(y)\right\| \leq L_{2}\|x-y\|
$$

for all $x, y \in \Omega, k \in \mathbb{Z}$.

Assume that

$$
\frac{K_{0} L_{1}}{\omega(1+\underline{\mu \omega})}+\frac{K_{0} L_{2}}{1-e_{\ominus \omega}\left(\theta, t_{0}\right)}<1,
$$

then Equation (1) has a unique weighted piecewise pseudo S-almost automorphic mild solution.

Proof. Consider the nonlinear operator $\Gamma$ given by

$$
\Gamma \varphi=\int_{-\infty}^{t} T(t, s) f(s, \varphi(s)) \Delta s+\sum_{t_{k}<t} T\left(t, t_{k}\right) I_{k}\left(\varphi\left(t_{k}\right)\right) .
$$

By Theorem 7 , we see that $\Gamma$ maps $W P A A_{S}(\mathbb{T}, \rho)$ into $W P A A_{S}(\mathbb{T}, \rho)$.

It suffices now to show that the operator $\Gamma$ has a fixed point in $W P A A_{S}(\mathbb{T}, \rho)$. For $\varphi_{1}, \varphi_{2} \in$ $W P A A_{S}(\mathbb{T}, \rho)$, one has the following:

$$
\begin{aligned}
& \left\|\Gamma \varphi_{1}(t)-\Gamma \varphi_{2}(t)\right\|=\| \int_{-\infty}^{t} T(t, s)\left[f\left(s, \varphi_{1}(s)\right)-f\left(s, \varphi_{2}(s)\right)\right] \Delta s \\
& +\sum_{t_{k}<t} T\left(t, t_{k}\right)\left[I_{k}\left(\varphi_{1}\left(t_{k}\right)\right)-I_{k}\left(\varphi_{2}\left(t_{k}\right)\right)\right] \| \\
\leq & \int_{-\infty}^{t} \frac{K_{0}}{1+\mu(t) \omega} e_{\ominus \omega}(t, s)\left\|f\left(s, \varphi_{1}(s)\right)-f\left(s, \varphi_{2}(s)\right)\right\| \Delta s \\
& +\sum_{t_{k}<t} \frac{K_{0}}{1+\mu(t) \omega} e_{\ominus \omega}\left(t, t_{k}\right)\left\|I_{k}\left(\varphi_{1}\left(t_{k}\right)\right)-I_{k}\left(\varphi_{2}\left(t_{k}\right)\right)\right\| \\
= & \int_{-\infty}^{t}\left(-\frac{K_{0}}{\omega(1+\mu(t) \omega)}\right)(\ominus \omega) e_{\ominus \omega}(t, \sigma(s)) L_{1}\left\|\varphi_{1}(s)-\varphi_{2}(s)\right\| \Delta s \\
& +\sum_{t_{k}<t} \frac{K_{0}}{1+\mu(t) \omega} e_{\ominus \omega}\left(t, t_{k}\right) L_{2}\left\|\varphi_{1}\left(t_{k}\right)-\varphi_{2}\left(t_{k}\right)\right\| \\
\leq \quad & {\left[\frac{K_{0} L_{1}}{\omega(1+\mu \omega)}+\frac{K_{0} L_{2}}{1-e_{\ominus \omega}\left(\theta, t_{0}\right)}\right]\left\|\varphi_{1}-\varphi_{2}\right\| . }
\end{aligned}
$$


Since $\frac{K_{0} L_{1}}{\omega(1+\mu \omega)}+\frac{K_{0} L_{2}}{1-e_{\ominus} \omega\left(\theta, t_{0}\right)}<1, \Gamma$ is a contradiction. Hence, $\Gamma$ has a fixed point in $W P A A_{S}(\mathbb{T}, \rho)$, then Equation $(\overline{1})$ has a unique weighted piecewise pseudo $S$-almost automorphic mild solution. This completes the proof.

\section{Applications}

In this section, three examples are demonstrated as the applications of our obtained results.

Example 4. Let $\mathbb{T}_{1}$ be S-CCTS under shifts $\delta_{ \pm}$and $u: \mathbb{T}_{1} \times \mathbb{T}_{2} \rightarrow \mathbb{R}$, where the hybrid domain $\mathbb{T}_{1}$ is the time scale in Example (1) and $\mathbb{T}_{2}$ is a discrete time scale with a forward jump operator $\sigma_{2}$ and $0, \pi \in \mathbb{T}_{2}$, i.e.,

$$
\mathbb{T}_{1}=\overline{(-q)^{\mathbb{Z}}}=\left\{(-q)^{n}: q>1, n \in \mathbb{Z}\right\} \cup\{0\}, \text { where } q=\sqrt{3} .
$$

Consider the following system:

$$
\left\{\begin{array}{l}
\frac{u\left(q_{t} t, x\right)-u(t, x)}{\left(q_{t}-1\right) t}=\frac{\mu(x) u\left(q_{t}, t \sigma_{2}^{2}(x)\right)-(\mu(x)+\mu(\sigma(x))) u\left(q_{t} t, \sigma_{2}(x)\right)+\mu(\sigma(x)) u\left(q_{t} t, x\right)}{\mu(\sigma(x)) \mu^{2}(x)}+\frac{1}{18}(\sin \sqrt{2} t+g(t)) \cos u(t, x), \\
\quad t \in \mathbb{T}_{1}, t \neq t_{k}, x \in[0, \pi]_{\mathbb{T}_{2}} \\
\tilde{\Delta} u\left(t_{k}, x\right)=\beta_{k} u\left(t_{k}, x\right), k \in \mathbb{Z}, x \in[0, \pi]_{\mathbb{T}_{2}} \\
u(t, 0)=u(t, \pi)=0, t \in \mathbb{T}_{1},
\end{array}\right.
$$

where $g \in U P C\left(\mathbb{T}_{1}, \mathbb{R}\right)$ satisfies $|g(t)| \leq 1,\left(t \in \mathbb{T}_{1}\right)$ and $\rho(t)=|\sin t|+1, \beta_{k}=\frac{1}{450}(\sin k+\cos \sqrt{2} k+$ $g(k))$ and $t_{k}=q^{6 k}, t_{-k}=(-q)^{6 k+1}, k \in \mathbb{Z}^{+} \cup\{0\}$.

Define $\mathbb{X}=L^{2}[0, \pi]_{\mathbb{T}_{2}}$, let

$$
A u=\frac{\mu(x) u\left(q_{t} t, \sigma_{2}^{2}(x)\right)-(\mu(x)+\mu(\sigma(x))) u\left(q_{t} t, \sigma_{2}(x)\right)+\mu(\sigma(x)) u\left(q_{t} t, x\right)}{\mu(\sigma(x)) \mu^{2}(x)},
$$

where $u \in D(A)=H_{0}^{1}[0, \pi]_{\mathbb{T}_{2}} \cap H^{2}[0, \pi]_{\mathbb{T}_{2}}$. Clearly, it follows from the same discussion as Section 3.1 in [22], one can easily observe that the evolution system $\{T(t, s): t \geq s\}$ satisfies $\|T(t, s)\| \leq$ $\frac{1 / 2}{1+\mu(t)(1 / 2)} e_{\ominus \frac{1}{2}}(t, s)(t \geq s)$ with $K_{0}=1 / 2, \omega=1 / 2$. Furthermore, we obtain that $\left\{t_{k}^{j}\right\}=$ $\left\{\delta_{-}\left(t_{k}, t_{k+j}\right)\right\} \subset\left\{t_{k}\right\}, k, j \in \mathbb{Z}$, by Definition 3 , we can obtain $\left\{t_{k}^{j}\right\}, k, j \in \mathbb{Z}$, is an equipotentially S-almost automorphic sequence and for $k \in \mathbb{Z}^{+}$, we have

$$
t_{k}^{1}=\delta_{-}\left(t_{k}, t_{k+1}\right)=\frac{t_{k+1}}{t_{k}}=q^{6}, t_{-k}^{1}=\delta_{-}\left(t_{-k}, t_{-k-1}\right)=\frac{t_{-k-1}}{t_{-k}}=\frac{1}{q^{6}} .
$$

Let $f(t, u)=\frac{1}{18}(\sin \sqrt{2} t+g(t)) \cos u, I_{k}(u)=\beta_{k} u$. Noticing that $f$ and $I_{k}$ satisfy the assumptions given in Theorem 8 with $L_{1}=\frac{1}{3}$ and $L_{2}=\frac{1}{300}$, we obtain

$$
\frac{K_{0} L_{1}}{\omega(1+\underline{\mu} \omega)}+\frac{K_{0} L_{2}}{1-e_{\ominus \omega}(\theta, 1)}=\frac{K_{0} L_{1}}{\omega}+\frac{K_{0} L_{2}}{1-\sup _{k} \prod_{s \in\left[t_{k}, t_{k+1}\right)}\left(1-\frac{\sqrt{3}-1}{1+q^{2}} s\right)} \leq \frac{1}{3}<1 .
$$

In fact, noting that

$$
\frac{K_{0} L_{2}}{1-\sup _{k} \prod_{s \in\left[t_{k}, t_{k+1}\right)}\left(1-\frac{\sqrt{3}-1}{1+q^{2}} s\right)}=0
$$

since that

$$
\prod_{s \in\left[t_{k}, t_{k+1}\right)}\left(1-\frac{\sqrt{3}-1}{1+q^{2}} s\right) \rightarrow \infty \text { as } k \rightarrow \infty .
$$

Therefore, Equation (15) has a weighted piecewise pseudo S-almost automorphic mild solution. 
Example 5. Let $\mathbb{T}_{1}$ be S-CCTS under shifts $\delta_{ \pm}$and $u: \mathbb{T}_{1} \times \mathbb{T}_{2} \rightarrow \mathbb{R}$, where the time scale $\mathbb{T}_{1}=h \mathbb{Z}, h>0$ and $\mathbb{T}_{2}=\left\{q^{n}: q>1, n \in \mathbb{Z}\right\} \cup\{0, \pi\}$. Consider the following dynamic equation:

$$
\left\{\begin{array}{l}
\frac{u(t+h, x)-u(t, x)}{h}=\frac{u\left(t+h, q^{2} x\right)-(q+1) u(t+h, q x)+q u(t+h, x)}{q(q-1)^{2} x^{2}}+\frac{1}{18} g(t) \cos \sqrt{2} t \cos u(t, x), \\
\quad t \in \mathbb{T}_{1}, t \neq t_{k}, x \in[0, \pi]_{\mathbb{T}_{2}} \\
\tilde{\Delta} u\left(t_{k}, x\right)=\beta_{k} u\left(t_{k}, x\right), \quad k \in \mathbb{Z}, x \in[0, \pi]_{\mathbb{T}_{2}} \\
u(t, 0)=u(t, \pi)=0, t \in \mathbb{T}_{1}
\end{array}\right.
$$

where $g \in U P C\left(\mathbb{T}_{1}, \mathbb{R}\right)$ satisfies $|g(t)| \leq 1,\left(t \in \mathbb{T}_{1}\right)$ and $\rho(t)=e^{-t}+1, \beta_{k}=\frac{1}{17} g(k)$ and $t_{k}=4 k h, t_{-k}=$ $-4 k h, k \in \mathbb{Z}^{+} \cup\{0\}$.

Define $\mathbb{X}=L^{2}[0, \pi]_{\mathbb{T}_{2}}$ let

$$
A u=\frac{u\left(t+h, q^{2} x\right)-(q+1) u(t+h, q x)+q u(t+h, x)}{q(q-1)^{2} x^{2}}, u \in D(A)=H_{0}^{1}[0, \pi]_{\mathbb{T}_{2}} \cap H^{2}[0, \pi]_{\mathbb{T}_{2}} .
$$

Clearly, through Section 3.1 in [22], we have the evolution system $\{T(t, s): t \geq s\}$ that satisfies $\|T(t, s)\| \leq \frac{1 / 2}{1+\mu(t)(1 / 2)} e_{\ominus \frac{1}{2}}(t, s)(t \geq s)$ with $K_{0}=1 / 2, \omega=1 / 2$. Moreover, we have $\left\{t_{k}^{j}\right\}=\left\{t_{k+j}-t_{k}\right\} \subset$ $\left\{t_{k}\right\}, k, j \in \mathbb{Z}$, then $\left\{t_{k}^{j}\right\}, k, j \in \mathbb{Z}$, is an equipotentially S-almost automorphic sequence. For $k \in \mathbb{Z}^{+}$,

$$
t_{k}^{1}=t_{k+1}-t_{k}=4 h=t_{-k-1}-t_{-k} .
$$

Hence, $\theta=\inf _{k \in \mathbb{Z}}\left(t_{k+1}-t_{k}\right)=4 h>0$. Let $f(t, u)=\frac{1}{18} g(t) \cos \sqrt{2} t \cos u, I_{k}(u)=\beta_{k} u$. Clearly, both $f$ and $I_{k}$ satisfy the assumptions given in Theorem 8 with $L_{1}=\frac{1}{18}, L_{2}=\frac{1}{17}$. Moreover,

$$
\frac{K_{0} L_{1}}{\omega(1+\underline{\mu \omega})}+\frac{K_{0} L_{2}}{1-e_{\ominus}(\theta, 1)}=\frac{K_{0} L_{1}}{\omega(1+h \omega)}+\frac{K_{0} L_{2}}{\sup _{k}\left(1-\frac{1}{2+h}\right)^{4}}<\frac{1}{18(2+h)}+\frac{8}{17}<1 .
$$

Therefore, Equation (16) has a weighted piecewise pseudo S-almost automorphic mild solution.

Example 6. Let $\mathbb{T}_{1}$ be S-CCTS under shifts $\delta_{ \pm}$and $u: \mathbb{T}_{1} \times \mathbb{T}_{2} \rightarrow \mathbb{R}$, where the hybrid domain $\mathbb{T}_{1}$ is the time scale $\mathbb{N}_{0}^{2}$ and $\mathbb{T}_{2}=h \mathbb{Z} \cup\{0, \pi\}$. Consider the following dynamic equation:

$$
\left\{\begin{array}{l}
\frac{u\left((\sqrt{t}+1)^{2}, x\right)-u(t, x)}{2 \sqrt{t}+1}=\frac{u\left((\sqrt{t}+1)^{2}, x+2 h\right)-2 u\left((\sqrt{t}+1)^{2}, x+h\right)+u(x)}{h^{2}}+\frac{1}{21}(\sin t+\cos \sqrt{2} t+g(t)) \cos u(t, x), \\
\quad t \in \mathbb{T}_{1}, t \neq t_{k}, x \in[0, \pi]_{\mathbb{T}_{2}} \\
\tilde{\Delta} u\left(t_{k}, x\right)=\beta_{k} u\left(t_{k}, x\right), k \in \mathbb{Z}, x \in[0, \pi]_{\mathbb{T}_{2}} \\
u(t, 0)=u(t, \pi)=0, t \in \mathbb{T}_{1}
\end{array}\right.
$$

where $g \in U P C\left(\mathbb{T}_{1}, \mathbb{R}\right)$ satisfies $|g(t)| \leq 1,\left(t \in \mathbb{T}_{1}\right)$ and $\rho(t)=|\sin t|+1, \beta_{k}=\frac{1}{21}(\sin k+\cos \sqrt{2} k+$ $g(k))$ and $t_{k}=(k+3)^{2}, k \in \mathbb{Z}^{+} \cup\{0\}$.

Define $\mathbb{X}=L^{2}[0, \pi]_{\mathbb{T}_{2}}$, let

$$
A u=\frac{u\left((\sqrt{t}+1)^{2}, x+2 h\right)-2 u\left((\sqrt{t}+1)^{2}, x+h\right)+u(x)}{h^{2}}, u \in D(A)=H_{0}^{1}[0, \pi]_{\mathbb{T}_{2}} \cap H^{2}[0, \pi]_{\mathbb{T}_{2}} .
$$

Clearly, it follows from the same discussion as Section 3.1 in [22], one can easily observe that the evolution system $\{T(t, s): t \geq s\}$ satisfies $\|T(t, s)\| \leq \frac{1 / 2}{1+\mu(t)(1 / 2)} e_{\odot \frac{1}{2}}(t, s)(t \geq s)$ with $K_{0}=1 / 2, \omega=1 / 2$. In addition, we obtain an equipotentially S-almost automorphic sequence $\left\{t_{k}^{j}\right\}=\left\{\delta_{-}\left(t_{k}, t_{k+j}\right)\right\} \subset\left\{t_{k}\right\}, k, j \in \mathbb{Z}$, with

$$
t_{k}^{1}=\delta_{-}\left(t_{k}, t_{k+1}\right)=\left(\sqrt{t_{k+1}}-\sqrt{t_{k}}\right)^{2}=1
$$


Hence, $\theta=\inf _{k \in \mathbb{Z}}\left(\delta_{-}\left(t_{k}, t_{k+1}\right)\right)=1$. Let $f(t, u)=\frac{1}{21}(\sin t+\cos \sqrt{2} t+g(t)) \cos u, I_{k}(u)=\beta_{k} u$. Clearly, both $f$ and $I_{k}$ satisfy the assumptions given in Theorem 8 with $L_{1}=L_{2}=\frac{1}{6}$. Moreover,

$$
\frac{K_{0} L_{1}}{\omega(1+\underline{\mu \omega})}+\frac{K_{0} L_{2}}{1-e_{\ominus \omega}(\theta, 1)}<\frac{1}{6}+\frac{1 / 2}{6\left(1-e^{-\frac{1}{2}}\right)} \approx 0.3785<1
$$

Therefore, Equation (17) has a weighted piecewise pseudo S-almost automorphic mild solution.

\section{Conclusions and Open Problems}

In this paper, we have introduced a concept of complete-closed time scales attached with a shift direction under non-translational shifts (S-CCTS). This is the first attempt to introduce and study the concepts of $S$-equipotentially almost automorphic sequence, discontinuous $S$-almost automorphic functions and weighted piecewise pseudo $S$-almost automorphic functions. Then, we apply the introduced concepts to investigate the existence of weighted piecewise pseudo $S$-almost automorphic mild solutions for a class of evolution impulsive equations on hybrid domains. Finally, we apply the obtained results to $\Delta$-partial dynamic equations from which one can see the established results are feasible and effective for $q$-difference partial dynamic equations among others. It is obvious that these results are more general and comprehensive than previous literature.

On the other hand, by virtue of S-CCTS, the almost automorphic problems of $q$-difference partial dynamic equations can also be introduced and studied. In fact, from the construction of almost automorphy of functions in Section 3, one can also establish some new types of functions with almost automorphy. Moreover, by introducing the concepts of almost automorphic functions and $\Delta$-almost automorphic functions and developing an appropriate approach, we will study almost automorphic problems of $\Delta$-partial dynamic equations on irregular time scales, which will be the topic of our future work.

Based on our discussion, we introduce the following open problems:

(1) For the highly hybrid time scales such as $\mathbb{T}=\overline{q^{\mathbb{Z}}} \cup\{h \mathbb{Z}\} \cup \mathbb{N}_{0}^{\frac{1}{2}}$, how to provide an effective way to construct the shift operators?

(2) How to establish a feasible method to study the almost automorphy of solutions to discontinuous dynamic systems on highly hybrid time scales?

Author Contributions: All authors contributed equally and significantly in this paper and typed, read, and approved the final manuscript. All authors have read and agreed to the published version of the manuscript.

Funding: This work is supported by NSFC (No. 11961077, 11601470), IRTSTYN and Joint Key Project of Yunnan Provincial Science and Technology Department of Yunnan University (No. 2018FY001(-014)) .

Conflicts of Interest: The authors declares no conflict of interest.

\section{References}

1. Bochner, S. Uniform convergence of monotone sequences of functions. Proc. Natl. Acad. Sci. USA 1961, 47, 582-585. [CrossRef]

2. Bochner, S. A new approach in almost-periodicity. Proc. Natl. Acad. Sci. USA 1962, 48, 2039-2043. [CrossRef]

3. Bochner, S. Continuous mappings of almost automorphic and almost periodic functions. Proc. Natl. Acad. Sci. USA 1964, 52, 907-910. [CrossRef] [PubMed]

4. Chang, Y.K.; Zhao, Z.H.; Nieto, J.J. Pseudo almost automorphic and weighted pseudo almost automorphic mild solutions to semi-linear differential equations in Hilbert spaces. Rev. Mater. Complut. 2011, 24, 421-438. [CrossRef]

5. Chang, Y.K.; Zhang, R.; N'Guérékata, G.M. Weighted pseudo almost automorphic solutions to nonautonomous semilinear evolution equations with delay and $S^{p}$-weighted pseudo almost automorphic coefficients. Topol. Methods Nonlinear Anal. 2014, 43, 69-88. [CrossRef]

6. Chang, Y.K.; Feng, T.W. Properties on measure pseudo almost automorphic functions and applications to fractional differential equations in Banach spaces. Electr. J. Differ. Equ. 2018, 47, 1-14. 
7. Ding, H.S.; Liang, J.; N'Guérékata, G.M.; Xiao, T.J. Existence of positive almost automorphic solutions to neutral nonlinear integral equations. Nonlinear Anal. Theory Methods Appl. 2008, 69, 1188-1199. [CrossRef]

8. Ding, H.S.; Liu, Q.L.; Nieto, J.J. Existence of positive almost periodic solutions to a class of hematopoiesis model. Appl. Math. Model. 2016, 40, 3289-3297. [CrossRef]

9. Diagana, T. Existence of globally attracting almost automorphic solutions to some nonautonomous higher-order difference equations. Appl. Math. Comput. 2013, 219, 6510-6519. [CrossRef]

10. Diagana, T. Almost Automorphic Type and Almost Periodic Type Functions in Abstract Spaces; Developments in Mathematics Series; Springer International Publishing: Cham, Switzerland, 2013; ISBN 978-3-319-00848-6.

11. N'Guérékata, G.M. Topics in Almost Automorphy; Springer: Berlin, Germany , 2005.

12. N'Guérékata, G.M. Almost Automorphic and Almost Periodic Functions in Abstract Spaces; Kluwer Academic/Plenum Publishers: New York, NY, USA, 2001.

13. Hilger, S. Ein Maßkettenkalkül mit Anwendung auf Zentrumsmannigfaltigkeiten. Ph.D. Thesis, Universität Würzburg, Würzburg, Germany, 1989.

14. Bohner, M.; Peterson, A. Dynamic Equations on Time Scales; Birkhauser: Boston, MA, USA, 2001.

15. Guseinov, G.S. Integration on time scales. J. Math. Anal. Appl. 2003, 285, 107-127. [CrossRef]

16. Agarwal, R.P.; O'Regan, D. Some comments and notes on almost periodic functions and changing-periodic time scales. Electr. J. Math. Anal. Appl. 2018, 6, 125-136.

17. Agarwal, R.P.; Bohner, M.; O’Regan, D.; Peterson, A. Dynamic equations on time scales: A survey. J. Comput. Appl. Math. 2006, 285, 1-26. [CrossRef]

18. Akhmet, M.U.; Turan, M. The differential equations on time scales through impulsive differential equations. Nonlinear Anal. Theory Methods Appl. 2006, 65, 2043-2060. [CrossRef]

19. Akhmet, M.U.; Turan, M. Differential equations on variable time scales. Nonlinear Anal. Theory Methods Appl. 2009, 70, 1175-1192. [CrossRef]

20. Akhmet, M.U.; Beklioglu, M.; Ergenc, T.; Tkachenko, V.I. An impulsive ratio-dependent predator-prey system with diffusion. Nonlinear Anal. Real World Appl. 2006, 7, 1255-1267. [CrossRef]

21. Bohner, M.; Streipert, S.; Torres, D.F.M. Exact solution to a dynamic SIR model. Nonlinear Anal. Hybrid Syst. 2019, 32, 228-238. [CrossRef]

22. Jackson, B. Partial dynamic equations on time scales. J. Comput. Appl. Math. 2006, 186, 391-415. [CrossRef]

23. Martynyuk, A.A.; Stamova, I.M.; Martynyuk-Chernienko, Y.A. Matrix Lyapunov functions method for sets of dynamic equations on time scales. Nonlinear Anal. Hybrid Syst. 2019, 34, 166-178. [CrossRef]

24. Martynyuk, A.A.; Stamova, I.M. Stability of sets of hybrid dynamical systems with aftereffect. Nonlinear Anal. Hybrid Syst. 2019, 32, 106-114. [CrossRef]

25. Aydogan, M.S.; Baleanu, D.; Mousalou, A.; Rezapour, S. On high order fractional integro-differential equations including the Caputo-Fabrizio derivative. Bound. Value Probl. 2018, 15, 90. [CrossRef]

26. Baleanu, D.; Mousalou, A.; Rezapour, S. On the existence of solutions for some infinite coefficient-symmetric Caputo-Fabrizio fractional integrodifferential equations. Bound. Value Probl. 2017, 9, 145. [CrossRef]

27. Mozyrska, D.; Torres, D.F.M.; Wyrwas, M. Solutions of systems with the Caputo-Fabrizio fractional delta derivative on time scales. Nonlinear Anal. Hybrid Syst. 2019, 32, 168-176. [CrossRef]

28. Ortigueira, M.D.; Torres, D.F.M.; Trujillo, J.J. Exponentials and Laplace transforms on nonuniform time scales. Commun. Nonlinear Sci. Numer. Simul. 2016, 39, 252-270. [CrossRef]

29. Hong, S.; Peng, Y. Almost periodicity of set-valued functions and set dynamic equations on time scales. Inform. Sci. 2016, 330, 157-174. [CrossRef]

30. Kéré, M.; N'Guérékata, G.M. Almost automorphic dynamic systems on time scales. Panam. Math. J. 2018, 28, 19-37.

31. Mophou, G.; N'Guérékata, G.M.; Milcé, A. Almost automorphic functions of order $n$ and applications to dynamic equations on time scales. Discret. Dyn. Nat. Soc. 2014, 2014, 410210. [CrossRef]

32. N'Guérékata, G.M.; Milcé, A.; Mado, J.C. Asymptotically almost automorphic functions of order $n$ and applications to dynamic equations on time scales. Nonlinear Stud. 2016 20, 305-322.

33. Wang, C.; Agarwal, R.P.; O'Regan, D.; Sakthivel, R. Theory of Translation Closedness for Time Scales-With Applications to Translation Functions and Dynamic Equations; Developments in Mathematics Series; Springer: Cham, Switzerland, 2020; Volume 62.

34. Adıvar, M.; Raffoul, Y.N. Existence of resolvent for Volterra integral equations on time scales. Bull. Aust. Math. Soc. 2006, 82, 139-155. [CrossRef] 
35. Adivar, M. A new periodic concept for time scales. Math. Slovaca 2006, 63, 817-828.

36. Wang, C.; Agarwal, R.P.; O'Regan, D. Calculus of fuzzy vector-valued functions and almost periodic fuzzy vector-valued functions on time scales. Fuzzy Sets Syst. 2019, 375, 1-52. [CrossRef]

37. Wang, C.; Agarwal, R.P.; Sakthivel, R. Almost periodic oscillations for delay impulsive stochastic Nicholson's blowflies timescale model. Comput. Appl. Math. 2018, 37, 3005-3026. [CrossRef]

38. Wang, C.; Agarwal, R.P. Almost automorphic functions on semigroups induced by complete-closed time scales and application to dynamic equations. Discret. Contin. Dyn. Syst. B 2020, 25, 781-798. [CrossRef]

39. Stamov, G.T. Almost Periodic Solutions of Impulsive Differential Equations; Lecture Notes in Mathematics; Springer: Berlin/Heidelberg, Germany, 2012.

40. Stamova, I.M. Stability Analysis of Impulsive Functional Differential Equations; de Gruyter Expositions in Mathematics; Walter de Gruyter GmbH \& Co. KG: Berlin, Germany, 2009.

41. Wang, C.; Agarwal, R.P. Uniformly rd-piecewise almost periodic functions with applications to the analysis of impulsive $\Delta$-dynamic system on time scales. Appl. Math. Comput. 2015, 259, 271-292.

42. Wang, C. Almost periodic solutions of impulsive BAM neural networks with variable delays on time scales. Commun. Nonlinear Sci. Numer. Simul. 2014, 19, 2828-2842. [CrossRef]

43. Wang, C.; Agarwal, R.P. Weighted piecewise pseudo almost automorphic functions with applications to abstract impulsive $\nabla$-dynamic equations on time scales. Adv. Differ. Equ. 2014, 153, 1-29. [CrossRef]

(C) 2020 by the authors. Licensee MDPI, Basel, Switzerland. This article is an open access article distributed under the terms and conditions of the Creative Commons Attribution (CC BY) license (http:/ / creativecommons.org/licenses/by/4.0/). 